\title{
New reproduction number and acceleration parameter introduced and applied to COVID-19
}

Eckhard Rebhan (D E-I.Rebhan@t-online.de)

Heinrich-Heine-Universitat Dusseldorf https://orcid.org/0000-0003-4224-4671

\section{Research Article}

Keywords: reproduction number, acceleration parameter, covid-19 pandemic, incidence,exponential growth

Posted Date: February 9th, 2021

DOI: https://doi.org/10.21203/rs.3.rs-224404/v1

License: (c) (i) This work is licensed under a Creative Commons Attribution 4.0 International License. Read Full License 


\title{
New reproduction number and acceleration parameter introduced and applied to covid-19
}

\section{E. Rebhan}

Received: date / Accepted: date

\begin{abstract}
To characterize the progression of a pandemic, a well interpretable reproduction number is introduced which is easily applicable to many different situations due to its handy analytical form. On the basis of its derivation it can be understood as a cross between a volatile instantaneous reproduction number and the more robust effective reproduction number commonly used. Starting from it, a further quantity, termed acceleration parameter, is introduced, which facilitates a more differentiated characterization of the infection dynamics. In particular, it enables the precise determination of when the limit to exponential growth is reached and exceeded. A variety of possible developments is examined, including linear and exponential growth of the infection numbers as well as sub- and super-exponential growth. It turned out useful to incorporate the incidence as a further epidemiological indicator. It is used for calculating the trace that the progression of the pandemic leaves behind on a plain spanned by itself and the acceleration parameter. This plane can be divided into a dangerous area, where the pandemic becomes uncontrollable, and a safer area that must be the target of mitigation efforts. At present, many countries and the world as a whole are mired in the dangerous area.
\end{abstract}

Keywords reproduction number $\cdot$ acceleration parameter $\cdot$ covid-19 pandemic . incidence $\cdot$ exponential growth

\section{Introduction}

In order to keep the health damage and death cases caused by the covid-19 pandemic as low as possible, significant restrictions and unfamiliar actions are expected of the population. For getting people to understand why these are necessary, it is important that the reasons for them are communicated as precise and intelligible as possible.

\section{E. Rebhan}

Institut für Theoretische Physik, Heinrich-Heine-Universität, D-40225 Düsseldorf, Germany E-mail: rebhan@thphy.uni-duesseldorf.de 
This in turn presupposes that the knowledge about the various aspects of the pandemic is as extensive as possible, while being precise and thorough. This article is written with the intention and hope that the results obtained will make a small contribution to this.

First, in Section 2 a new reproduction number $R_{Z}$ is introduced that has similarities with both a rather volatile instantaneous R-number [1] and the more common effective reproduction number [2], and can be understood as something in between. The latter can be integrated into our formalism and is compared with our $R_{Z}$ in Sections 5.1 and 5.2. Our $R_{Z}$ is based on a fit function obtained by a best least-squares fit to the discrete reported infection numbers. It has a very simple and easily interpretable mathematical form and can therefore rather effortlessly be applied to many diversified situations. A further much more comprehensive variable, referred as the acceleration parameter $A$, is then derived from an essential constituent of the latter in Section 3. It turns out to be particularly useful, because its values provide more differentiated information about the progression of the infection numbers. Both quantities are derived from the temporal progression $Z(t)$ of the accumulated number $Z$ of infected individuals, and the behavior of both is determined for a number of different infection scenarios. In Section 4 it is shown that, depending on the circumstances, unreported cases have no or only a minor impact on $R_{Z}(t)$ and $A(t)$. In Section 5 the two quantities are evaluated for the progression of the covid-19 pandemic in the world as a whole. Thereby a rather early and a more recent period of time are considered. Section 6 deals with a few scenarios that have occurred similarly or could occur in the future.

Like the common effective reproduction number, our new reproduction number $R_{Z}$ and even our acceleration parameter $A$ are not sufficient on their own for a complete characterization of the prevailing stage of the pandemic. For this reason, a function $I(t)$ for the cumulative incidence is derived in section 7, again using a best fit method. Thereafter it is investigated which trace the pandemic is leaving behind on a plane with the abscissa $I$ and the ordinate $A$ by entering in it the curve $A(I(t))$ obtained by the parametric plot $\{I(t), A(t)\}$.

In the concluding Section 8 in addition to a summary of the results obtained some qualitative properties of the pandemic are discussed.

\section{New reproduction number $\boldsymbol{R}_{z}$}

Reproduction number is a term used in epidemiology with the intention to indicate how many uninfected people are contaminated by one infected person on average. It depends on many circumstances and is generally difficult to determine, more or less inaccuracies having to be accepted. Accordingly, there are different definitions that are adapted to different situations or different demands on accuracy (see e.g. [3]). The basic reproduction number $R_{0}$ [4] applies to the first infected person in a population that is neither vaccinated nor otherwise protected. In this paper we are concerned with alternatives to the effective reproduction number $R_{t}$, which takes into account vaccinations, protective measures and immunity gained through conquered disease, and is therefore time-dependent. 
A very simple definition of the latter is

$$
R_{t}=\frac{E_{t}}{E_{t-4}}
$$

where $E_{t}$ is the number of newly infected individuals at the time $t$. Formally, $R_{t}$ can be interpreted in such a way that those newly infected at time $t$ were infected by those newly infected at time $t-4$. In fact, people who were newly infected before and after $t-4$ will also be involved, and those infected at $t-4$ will not all be involved, so that $E_{t-4}$ is more of a kind of mean. Because the daily number of newly infected fluctuates very strongly, the above $R_{t}$ is also very volatile and therefore hardly used. The definition

$$
R_{t, \tau}=\frac{\bar{E}_{t}^{\tau}}{\bar{E}_{t-4}^{\tau}} \quad \text { with } \quad \bar{E}_{t}^{\tau}=\frac{1}{\tau} \sum_{s=t-\tau+1}^{t} E_{s}, \quad \bar{E}_{t-4}^{\tau}=\frac{1}{\tau} \sum_{s=t-\tau+1}^{t} E_{s-4}
$$

involves mean values and proves to be much more robust. For $\tau$ the value 7 is often used, and the number 4 in $t-4$ and $s-4$ has to do with the time of highest infectiosity after an infection and has proven to be useful for covid-19.

\subsection{Definition of $R_{Z}(t)$}

For introducing our reproduction number $R_{Z}$, we assume that a list of successive pairs of values $\left\{t_{n}, Z_{n}\right\}, n=0,1,2, \ldots$ [5] is given, where $Z_{n}$ is the number of cumulative cases at time $t_{n}$ (total number of individuals known to have been infected by time $t_{n}$ ). In this paper the latter is given in multiples of the time unit day, so that $t$ constitutes a dimensionless variable. The time steps $t_{n}-t_{n-1}$ can be one or several days. For the pairs $\left\{t_{n}, Z_{n}\right\}$ a best least-squares fit function $Z(t)$ is constructed, which in many cases can be done using a power series $Z(t)=\sum_{0}^{N} c_{n} t^{n}$ [6]. (In this way, the data is smoothed, as is also done in the above calculation of $\left.R_{t, \tau}\right)$. The current increase $\left.\Delta Z\right|_{t}$ of $Z(t)$ is $\dot{Z}(t) \Delta t$ with $\dot{Z}(t)=d Z(t) / d t$, and the increase $\left.\Delta Z\right|_{t-\Delta t}$ of $Z(t)$ at the time $t-\Delta t$ is $\dot{Z}(t-\Delta t) \Delta t$. In analogy to Eqs. (1)-(2) we now define

$$
R_{Z}(t)=\frac{\left.\Delta Z\right|_{t}}{\left.\Delta Z\right|_{t-\Delta t}} .
$$

For sufficiently small $\Delta t$ we can use the truncated expansion

$$
\dot{Z}(t-\Delta t)=\dot{Z}(t)-\ddot{Z}(t) \Delta t,
$$

insert it in Eq. (3) and obtain

$$
R_{Z}(t)=\frac{\dot{Z}(t) \Delta t}{\dot{Z}(t-\Delta t) \Delta t}=\frac{\dot{Z}(t)}{\dot{Z}(t)-\ddot{Z}(t) \Delta t} .
$$

Because the number of infections in a pandemic is only recorded on a daily basis, the smallest time interval that can be meaningfully used in this context is 1 day. Therefore We put $\Delta t=1$, and with this after a slight reshaping we finally get

$$
R_{Z}(t)=\frac{1}{1-\ddot{Z}(t) / \dot{Z}(t)}=\frac{1}{1-\dot{V}_{Z}(t) / V_{Z}(t)} \quad \text { with } \quad V_{Z}(t)=\dot{Z}(t) .
$$


$V_{Z}(t)$ is the growth velocity of $Z(t)$. On cursory inspection, it looks like $R_{Z}(t)$ is an instantaneous value related only to the moment $t$ and is therefore as volatile as the $R_{t}$ of Eq. (1). Contrary to this, the calculation of the effective reproduction number $R_{t, \tau}$ from Eq. (2) includes also past values in the numerator, and the data are smoothed by averaging over several days. On closer inspection, however, this also applies to our $R_{Z}(t)$ because in determining $Z(t)$ via a best least-squares fit to a data set $\left\{t_{n}, Z_{n}\right\}$, past data are included as well as smoothing takes place.

With $R_{t, \tau}$, the value 1 is easy to understand because $\bar{E}_{t}^{\tau}=\bar{E}_{t-4}^{\tau}$ means, that the number of newly infected individuals does not change, or, in other words, that on average each infected individual infects only one other during its contagious stage. For the fact, that the values $\neq 1$ also indicate the number of individuals infected by a single person, a simple explanation is given after Eq. (22). Our $R_{Z}$ has a different interpretation and can be used for other purposes. According to Eq. (4) it quantifies the conditions under which it increases or decreases: The greater or smaller the relative acceleration $\dot{V}_{Z} / V_{Z}$, the larger or smaller it becomes. As with $R_{t, \tau}, R_{Z}$ equals 1 for constant $\dot{Z}(t)$. Incidentally, it would be possible to use the number

$$
Q(t)=\frac{\ddot{Z}(t)}{\dot{Z}(t)}=\frac{\dot{V}_{Z}(t)}{V_{Z}(t)}
$$

as another parameter for characterizing the state of the pandemic, because due to the biunique relation

$$
R_{Z}=\frac{1}{1-Q}
$$

between $R_{Z}$ and $Q$ following from Eqs. (4)-(5), both quantities have the same information content. However, in Section 3 it is shown that a modification of $Q(t)$ is a much better choice.

\subsection{Properties of $R_{Z}$}

Linear growth: The general math expression for linear growth of $Z(t)$ is

$$
Z(t)=Z_{0}+\alpha\left(t-t_{0}\right) \quad \text { with } \quad Z_{0}=Z\left(t_{0}\right) \quad \text { and } \quad \alpha>0 .
$$

Inserting the resulting relations $\dot{Z}(t)=\alpha$ and $\ddot{Z}(t)=0$ in Eqs. (4) or (5) yields

$$
R_{Z}=1 \quad \text { or } \quad Q=0
$$

Vice versa Eq. (7) follows from Eqs. (8). For the growth velocity we get $V_{Z}=\alpha=$ const . As with $R_{t, \tau}=1$, this means that during its infectious phase, each infected person infects exactly one other person who has not yet been infected. For large values of the slope $\alpha$, linear growth can also be very dangerous. 
Completely exponential growth: The general math expression for exponential growth of $Z(t)$ is

$$
Z(t)=Z_{0} \mathrm{e}^{\alpha\left(t-t_{0}\right)} \quad \text { with } \quad Z_{0}=Z\left(t_{0}\right) \quad \text { and } \quad \alpha>0
$$

Due to $\dot{Z}(t)=\alpha Z(t)$ and $\ddot{Z}(t)=\alpha^{2} Z(t)$ then $\dot{Z}(t)$ and $\ddot{Z}(t)$ also grow exponentially. For reasons that will soon become apparent, we call this type of growth completely exponential. According to Eq. (4) the corresponding value of $R_{Z}$ is

$$
R_{Z}=1 /(1-\alpha)>1
$$

Incomplete exponential growth: We now look at what happens with $Z(t)$, when $R_{Z}$ or, equivalently, $Q=\dot{V}_{Z}(t) / V_{Z}$ is kept constant. The immediate consequence of this is

$$
\dot{Z}(t)=V_{Z}(t)=V_{Z 0} \mathrm{e}^{Q\left(t-t_{0}\right)} \quad \text { and } \quad \dot{V}_{Z}(t)=Q V_{Z 0} \mathrm{e}^{Q\left(t-t_{0}\right)}
$$

Further integration of the first equation leads to

$$
Z(t)=Z_{0}-\left(V_{Z 0} / Q\right)+\left(V_{Z 0} / Q\right) \mathrm{e}^{Q\left(t-t_{0}\right)}
$$

The comparison with Eq. (9) shows that complete exponential growth is only obtained if $Q=V_{Z 0} / Z_{0}$. Otherwise we call growth where $V_{Z}(t)$ and $\dot{V}_{Z}(t)$ grow exponentially, but $Z(t)$ does not, as incompletely exponential.

Growth of general $V_{Z}(t)$ : The behavior of a general $V_{Z}(t)$ can be classified by comparison with exponential functions. For this purpose we consider $V_{Z}\left(t_{0}\right)=V_{Z 0}$ and $\dot{V}_{Z}\left(t_{0}\right)=a_{0}$ as given and compare the progression of $V_{Z}(t)$ at $t=t_{0}$ with that of an exponential function $V_{Z 0} \mathrm{e}^{\alpha\left(t-t_{0}\right)}$ running through the same point $V_{Z 0}$ with the same slope $a_{0}$. (This connection between the exponential function and $V_{Z}(t)$ is important, because otherwise there would be absolute arbitrariness with regard to functions to be used for comparison.) From the last condition results $a_{0}=\alpha V_{Z 0}$ or

$$
\alpha=a_{0} / V_{Z 0}=Q\left(t_{0}\right)=Q_{0},
$$

where Eq. (5) was used last; so $V_{Z}(t)$ behaves at $t=t_{0}$ like $V_{Z 0} \mathrm{e}^{Q_{0}\left(t-t_{0}\right)}$. Omitting the index 0 and using Eq. (6), in regard to the local growth of $V_{Z}(t)$ (i.e. the growth at the time $t$ ) we can draw the conclusions

$$
V_{Z}(t)\left\{\begin{aligned}
\text { grows exponentially for } Q(t)>0 & \text { or } R_{Z}>1 \\
\text { stagnates for } Q(t)=0 & \text { or } R_{Z}=1 \\
\text { decays exponentially for } Q(t)<0 & \text { or } R_{Z}<1
\end{aligned}\right.
$$

the rate of growth or decay being $\alpha=Q(t)$. 
Table 1 Local behavior of $V_{Z}(t) / V_{Z 0}$ and $Z(t) / Z_{0}$ with use of the abbreviations $Q_{0}=V_{Z 0} / Z_{0}$ and $R_{Z 0}=1 /\left(1-Q_{0}\right)$. In the 1 st and 3rd line the growth of $V_{Z}(t) / V_{Z 0}$ is exponential. $V_{Z}(t) / V_{Z 0}$ and $Z(t) / Z_{0}$ behave differently in some ranges.

\begin{tabular}{l|l|c|c}
\hline & & $V_{Z}(t) / V_{Z 0}$ & $Z(t) / Z_{0}$ \\
\hline$Q>Q_{0}$ & $R_{Z}>R_{Z 0}$ & faster than $Z(t) / Z_{0}$ & slower than $V_{Z} / V_{Z 0}$ \\
$Q=Q_{0}$ & $R_{Z}=R_{Z 0}$ & completely exponential & completely exponential \\
$Q<Q_{0}$ & $R_{Z}<R_{Z 0}$ & slower than $Z(t) / Z_{0}$ & faster than $V_{Z} / V_{Z 0}$ \\
$Q=0$ & $R_{Z}=1$ & no growth & linear growth \\
$Q<0$ & $R_{Z}<1$ & exponential decay & slow growth \\
\hline
\end{tabular}

Growth of corresponding $Z(t)$ : We consider $Z(t)$ at a time $t=t_{0}+\delta t$ very close to $t_{0}$, i.e. with $\delta t / t_{0} \ll 1$. Up to first order in the small quantity $\delta t$ we have

$$
Z(t)=Z_{0}+\left.\dot{Z}(t)\right|_{t_{0}} \delta t=Z_{0}+V_{Z 0} \delta t .
$$

Now we compare the growth of $Z(t)$ with that of the function $Z_{e x}(t)=Z_{0} \mathrm{e}^{\alpha\left(t-t_{0}\right)}$ for $t-t_{0}=\delta t$, which up to terms of the second order is given by

$$
Z_{e x}(t)=Z_{0}(1+\alpha \delta t)
$$

The comparison with Eq. (15) shows that $Z(t)$ behaves like $Z_{e x}(t)$ for

$$
V_{Z 0} / Z_{0}=\alpha
$$

According to the last paragraph, $V_{Z}(t)$ behaves like $V_{Z 0} \mathrm{e}^{Q_{0}\left(t-t_{0}\right)}$, so that for small $t-t_{0}=\delta t$ we get

$$
V_{Z}(t)=V_{Z 0} \mathrm{e}^{Q_{0} \delta t}=V_{Z 0}\left(1+Q_{0} \delta t\right) .
$$

From Eqs. (13) and (15)-(18) it follows by comparing $V_{Z}(t) / V_{Z 0}$ and $Z(t) / Z_{0}$

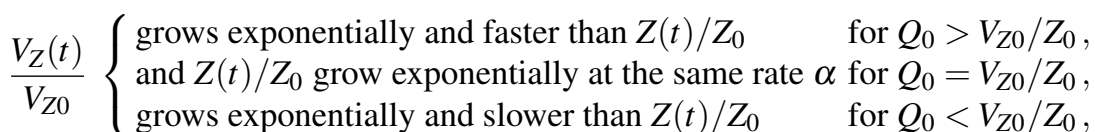

where the exponential growth of $V_{Z}(t) / V_{Z 0}$ takes place with the rate

$$
\alpha=Q_{0}=\frac{a_{0}}{V_{Z 0}} .
$$

For later purposes it should be noted that the conditions put on $Q_{0}$ in the array (19) can be rewritten in the form

$$
\frac{Z_{0} a_{0}}{V_{Z 0}^{2}}>=<1
$$

Summary: The results obtained for the local growth of $V_{Z}(t) / V_{Z 0}$ and $Z(t) / Z_{0}$ are listed in Table 1. 
2.3 Effective reproduction number $R_{t, \tau}$ in our notation

It cannot be expected that our $R_{Z}$ agrees with the effective reproduction number $R_{t, \tau}$, but it should at least qualitatively show a similar behavior. For this reason, let's look at how the $R_{t, \tau}$ from Eq. (2) is expressed in our approach in order to compare it with $R_{Z}$ later on. $\dot{Z}(t)$ corresponds to $E_{s}$, and the sum in the definition of $\bar{E}_{t}^{\tau}$ must be replaced by an integral, i.e. we have the replacement

$$
\bar{E}_{t}^{\tau} \rightarrow \frac{1}{\tau} \int_{t-\tau}^{t} \dot{Z}(s) d s=\frac{1}{\tau}[Z(t)-Z(t-\tau)]
$$

With this, we finally get

$$
R_{t, \tau}=\frac{Z(t)-Z(t-\tau)}{Z(t-4)-Z(t-4-\tau)}
$$

The numerator is the number of individuals who were infected in the time interval $[t-\tau, t]$, and the denominator is the same for a 4 days earlier time interval of equal duration $\tau$. The latter can roughly be viewed as the number of those who are responsible for the contamination of the newly infected in the interval $[t-\tau, t]$. This explains the interpretation given in the paragraph before Eq. (5). A comparison of $R_{Z}$ with $R_{t, \tau}$ is carried out in Section 5, and a particularly simple representation of $R_{t, \tau}$ is presented in Section 7.1.

\section{Acceleration parameter $A(t)$}

The terms linear growth and exponential growth are customarily used to describe different degrees in the dangerousness of the infection progress. The usual effective reproduction number $R_{t, \tau}$ ensures precisely linear growth for the value 1 . Values $>1$ are often viewed as evidence of exponential growth, but it is unclear from which value this applies. In this respect, our $R_{Z}$ allows more precise conclusions according to Table 1 . For further reaching consequences, however, both parameters are not fully satisfactory.

In the following, another quantity is proposed that enables more differentiated and precise assessments. It consists in a modification of $Q=\ddot{Z} / \dot{Z}$ introduced in Eq. (5) and is

$$
A(t)=\frac{Z(t) \ddot{Z}(t)}{\dot{Z}^{2}(t)}=\frac{Z(t) \dot{V}_{z}(t)}{V_{z}^{2}(t)} \quad \text { or } \quad A(t)=\frac{Z(t) Q(t)}{\dot{Z}(t)} .
$$

The relationship between $A(t)$ and $Q(t)$ shows that $A(t)$ contains more information than $Q(t)$. Note that $A$ is a dimensionless number even if $t$ has a dimension, a fact that influenced its definition. In retrospect, it turned out that the same combination of quantities also occurred when investigating properties of our $R_{Z}$ (see Eq. (21)).

\subsection{Properties of $A(t)$}

Linear growth: In this case $Z(t)$ is given by Eq. (7) so that $\ddot{Z}(t)=0$ and $A(t) \equiv 0$. Conversely, Eq. (7) follows from $A(t) \equiv 0$. 


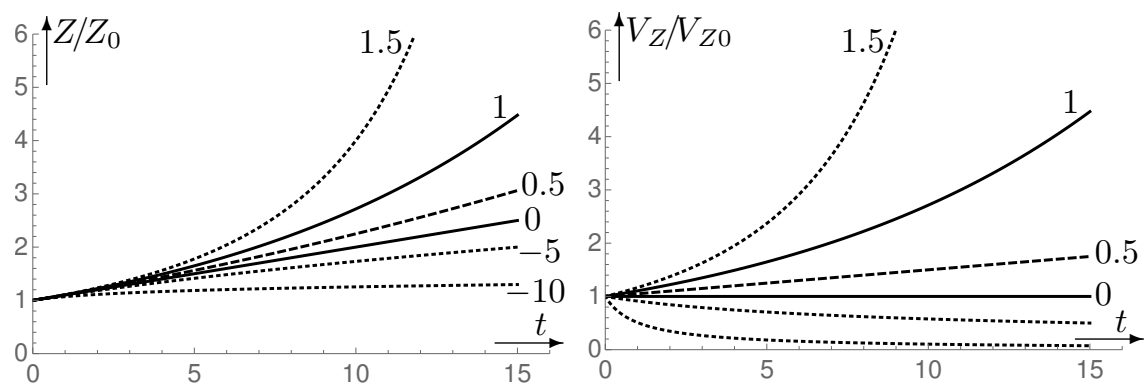

Fig. 1 The left diagram shows $Z(t) / Z_{0}$ and the right diagram $V_{Z}(t) / V_{Z 0}$ for $A=1.5,1,0.5,0,-5,-10$ in the order from top to bottom. The curves $A=1$ for exponential growth and $A=0$ for linear growth are solid. The dashed curve $A=0.5$ can (but must not) be considered as the boundary between super-linear and sub-exponential.

Complete exponential growth: In the case $Z(t)=Z_{0} \mathrm{e}^{\alpha\left(t-t_{0}\right)}$ of complete exponential growth we get $\dot{Z}(t)=\alpha Z, \ddot{Z}(t)=\alpha^{2} Z$ and $A(t) \equiv 1$, i.e. $A(t)$ assumes the same value 1 for all growth rates $\alpha$. Conversely, from $A(t) \equiv 1$ we get

$$
\frac{\ddot{Z}(t)}{\dot{Z}(t)}=\frac{\dot{V}_{Z}(t)}{V_{z}(t)}=\frac{d}{d t} \ln \frac{V_{Z}(t)}{V_{Z 0}}=\frac{\dot{Z}(t)}{Z(t)}=\frac{d}{d t} \ln \frac{Z(t)}{Z_{0}},
$$

from this by integration $Z_{0} V_{Z}(t) /\left(V_{Z 0} Z(t)\right)=1$, and with $V_{Z}(t)=\dot{Z}(t)$ and a further integration finally

$$
Z(t)=Z_{0} \mathrm{e}^{\alpha\left(t-t_{0}\right)} \quad \text { with } \quad \alpha=V_{Z 0} / Z_{0} .
$$

This means that the growth is completely exponential.

Incomplete exponential growth: From Eqs. (11)-(12) and (23b) ${ }^{1}$ we get after minor conversions

$$
A(t)=1+\left(\frac{Q Z_{0}}{V_{Z 0}}-1\right) \mathrm{e}^{-Q\left(t-t_{0}\right)}\left\{\begin{array}{lll}
>1 & \text { for } & Q>V_{Z 0} / Z_{0} \\
=1 & \text { for } & Q=V_{Z 0} / Z_{0} \\
<1 & \text { for } & Q<V_{Z 0} / Z_{0}
\end{array}\right.
$$

Growth with constant $A(t)=A$ : In this case a conversion of Eq. (23a) with use of $V_{Z}(t)=\dot{Z}(t)$ yields

$$
\frac{d}{d t} \ln \frac{V_{z}(t)}{V_{Z 0}}=\frac{\dot{V}_{z}(t)}{V_{z}(t)}=\frac{A \dot{Z}(t)}{Z(t)}=A \frac{d}{d t} \ln \frac{Z(t)}{Z_{0}} .
$$

Integrations with respect to $t$ yield $\ln \left(V_{z}(t) / V_{Z 0}\right)=A \ln \left(Z(t) / Z_{0}\right)$ or

$$
V_{Z}(t)=\dot{Z}(t)=V_{Z 0}\left(\frac{Z(t)}{Z_{0}}\right)^{A}
$$

\footnotetext{
${ }^{1}$ If in a numbered equation two equations are listed, the left is quoted with a and the right with $b$.
} 
and

$$
Z(t)=Z_{0}\left[1+\left(V_{Z 0} / Z_{0}\right)(1-A)\left(t-t_{0}\right)\right]^{1 /(1-A)} .
$$

From this we get $Z(t)=Z_{0}+V_{Z 0}\left(t-t_{0}\right)$ for $A=0$, i.e. $A=0$ corresponds to linear growth. For $A=1$ Eq. (26b) becomes $\dot{Z}(t)=\alpha Z(t)$ and yields $Z(t)=Z_{0} \mathrm{e}^{\alpha\left(t-t_{0}\right)}$ where $\alpha=V_{Z 0} / Z_{0}$. In Appendix 9 it is shown that for $A \rightarrow 1$ the same follows from Eq. (27).

Fig. 1 shows $Z(t) / Z_{0}$ (left plot) and $V_{Z}(t) / V_{Z 0}$ (right plot) for 6 different values of $A$ and a value of $V_{Z 0} / Z_{0}$ which is arbitrarily chosen but equal for all curves. From Eq. (26) follows immediately

$$
\frac{V_{Z}(t) / V_{Z 0}}{Z(t) / Z_{0}}=\left(\frac{Z(t)}{Z_{0}}\right)^{A-1}
$$

$Z(t)$ can only increase or at most stagnate, and in the acute phase of an epidemic, $Z(t)$ will definitely be be greater than $Z_{0}$ for $t>t_{0}$. It follows from this that

$$
\frac{V_{Z}(t)}{V_{Z 0}} \begin{cases}\text { grows faster than } Z(t) / Z_{0} & \text { for } A>1, \\ \text { grows equally fast as } Z(t) / Z_{0} & \text { for } A=1, \\ \text { grows slower than } Z(t) / Z_{0} & \text { for } A<1 .\end{cases}
$$

3.2 Classification of general growth by comparison with exponential growth

For the following it is assumed that $Z(t)$ is two times continuously differentiable.

Growth of a general $Z(t)$ : Expanding $Z(t)$ around an arbitrary time $t_{0}$ according to

$$
Z(t)=Z_{0}+V_{Z 0}\left(t-t_{0}\right)+a_{0}\left(t-t_{0}\right)^{2} / 2,
$$

with $\dot{Z}(t)=V_{Z 0}+a_{0}\left(t-t_{0}\right)$ and $\ddot{Z}(t)=a_{0}$ we get for $t \rightarrow t_{0}$

$$
A\left(t_{0}\right)=\frac{Z_{0} a_{0}}{V_{Z 0}^{2}} .
$$

The institutions that collect and process the information about the infections make public inter alia the daily number of new infections (daily cases), and the sum of them (cumulative cases), i.e. $V_{Z}(t)$ and $Z(t)$ in our analysis. Let us therefore consider different values $a_{0}$ for given values $Z_{0}$ and $V_{Z 0}$. In the case $Z(t)=Z_{0} \mathrm{e}^{\alpha\left(t-t_{0}\right)}$ we have $V_{Z 0}=\alpha Z_{0}, a_{0}=\alpha^{2} Z_{0}=V_{Z 0}^{2} / Z_{0}$ and from this, as expected, $A\left(t_{0}\right)=1$. For $a_{0}>V_{Z 0}^{2} / Z_{0}$ (i.e. $\left.A\left(t_{0}\right)>1\right)$, the curve $Z(t)$ is curved stronger upward than $Z_{0} \mathrm{e}^{\alpha\left(t-t_{0}\right)}$, and we therefore call it locally super-exponential. For $a_{0}<V_{Z 0}^{2} / Z_{0}$ (or $A\left(t_{0}\right)<1$ ), it is less curved whence we call it locally sub-exponential. For $a_{0}=0$ it is not curved, i.e. locally lin$e a r$, and for $a_{0}<0$ its growth is sub-linear (slower than linear) and stops growing at all for $V_{Z 0} \rightarrow 0$ with the consequence $A\left(t_{0}\right) \rightarrow-\infty$. These results are summarized in Table 2.

Obviously there are infinitely many different functions $Z(t)$ (e.g. polynomials), which run through the same point $Z_{0}$ with the same slope $\left.\dot{Z}(t)\right|_{t_{0}}=V_{Z 0}$ as the exponential function $Z(t)=Z_{0} \mathrm{e}^{\alpha\left(t-t_{0}\right)}$ used for comparison. A very simple example is 


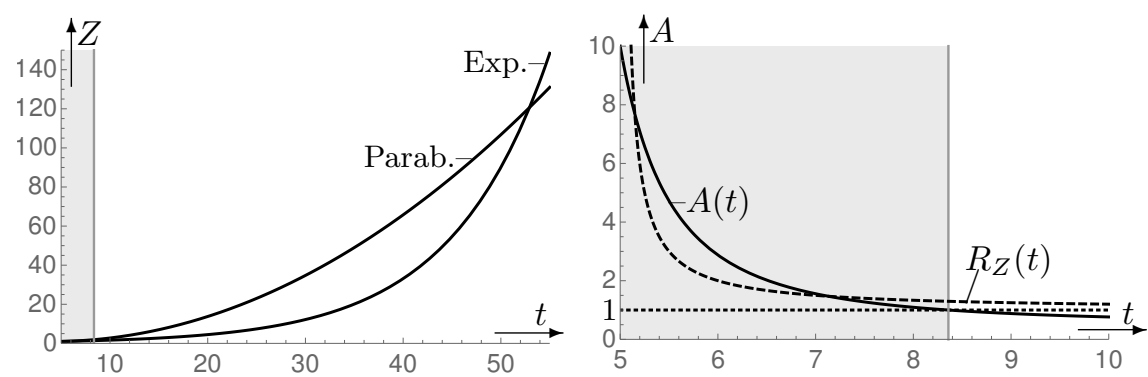

Fig. 2 The parabola and exponential function shown in the diagram on the left have the same function value and the same slope but different curvature at $t=0$. As a result, the parabola has higher values $Z(t)$ than the exponential function over a longer period of time. In the diagram on the right, the values $A(t)$ and $R_{Z}(t)$ belonging to the parabola are plotted, with the super- exponential area being shaded.

provided by the function $Z(t)$ in Eq. (30), a parabola, which, with the same values $Z_{0}$, $V_{Z 0}$ and sufficiently large $a_{0}$, grows over a large time interval faster than $Z_{0} \mathrm{e}^{\alpha\left(t-t_{0}\right)}$ (see Fig. 2). The comparison with exponential functions, on which the entries of Table 2 are based, is therefore not objectively prescribed but a choice made for reasons of utility and customariness. ${ }^{2}$ This situation has similarities e.g. with the evaluation of lengths (or temperatures), which can be measured in meters or yards (degrees Celsius or Fahrenheit). For reasons of continuity, the conditions of Table 2 not placed next to an equal sign are not only present locally, but in coherent time intervals of finite size.

Growth of a general $V_{Z}(t)$ : We also classify the behavior of $V_{Z}(t)$ by comparison with exponential functions. This time we consider the values $V_{Z}\left(t_{0}\right)=V_{Z 0}$ and $\dot{V}_{Z}\left(t_{0}\right)=a_{0}$ as given and compare the behavior with that of the function $V_{e x}(t)=V_{Z 0} \mathrm{e}^{\alpha\left(t-t_{0}\right)}$ running through the same point $V_{Z 0}$ with the same slope $a_{0}$, whereby for the latter the condition

$$
a_{0}=\alpha V_{Z 0}
$$

must be met. With this, it follows from Eq. (23) that $A_{0}=A\left(t_{0}\right)=\alpha /\left(V_{Z 0} / Z_{0}\right)$ or

$$
\alpha=A_{0}\left(V_{Z 0} / Z_{0}\right) \text {. }
$$

Since this implies $\alpha=V_{Z 0} / Z_{0} \geq 0$ for $A_{0}=1$, we can rewrite this result in the form

$$
\alpha\left(A_{0}\right)=\alpha(1) A_{0} .
$$

So we have the result that $V_{Z}(t) / V_{Z 0}$ behaves locally like $\mathrm{e}^{\alpha(1) A_{0}}$, i.e. grows exponentially for $A>0$, stagnates for $A_{0}=0$, and decays exponentially for $A_{0}<0$. (For reasons of space, this is not entered in Table 2.) It is shown in Appendix 10 that the result (28), derived for constant $A$, also applies locally to time-dependent $A$, what then also pertains to the statements in Eq. (29) derived from this. With omission of the subscript 0 , the results for the growth of $V_{Z}(t) / V_{Z 0}$ are also listed in Table 2.

\footnotetext{
2 One could argue that the exponential function $Z(t)=Z_{0} \mathrm{e}^{\alpha\left(t-t_{0}\right)}$ is particularly simple because it has only 2 parameters. However, the same is true for the parabola $Z_{0}\left(1+\alpha\left(t-t_{0}\right)+\alpha^{2}\left(t-t_{0}\right)^{2} / 2\right)$ which is obtained when the series expansion of the exponential function is terminated after the second order term.
} 
Table 2 Local behavior of $Z(t) / Z_{0}$ and $V_{Z}(t) / V_{Z 0}$. Defining $A(t)=0.5$ as the boundary between subexponential and super-linear is obvious but not mandatory. In the first 4 lines of the column for $V_{Z}(t) / V_{Z 0}$ the growth is exponential.

\begin{tabular}{l|l|l}
\hline \multicolumn{1}{c|}{$\mathrm{A}(\mathrm{t})$} & \multicolumn{1}{|c}{$Z(t) / Z_{0}$} & \multicolumn{1}{c}{$V_{Z}(t) / V_{Z 0}$} \\
\hline$A(t)>1$ & super-exponential growth & faster growth than $Z(t) / Z_{0}$ \\
$A(t)=1$ & exponential growth & same growth as $Z(t) / Z_{0}$ \\
$A(t) \in] 0.5,1[$ & sub-exponential growth & slower growth than $Z(t) / Z_{0}$ \\
$A(t) \in] 0,0.5]$ & super-linear growth & slower growth than $Z(t) / Z_{0}$ \\
$A(t)=0$ & linear growth & no growth \\
$A(t)<0$ & sub-linear growth & exponential decay \\
\hline
\end{tabular}

\section{Influence of unreported cases on $R_{z}(t)$ and $A(t)$}

In an epidemic or pandemic there is always a non-negligible number of infections which for various reasons are not included in the official census. Therefore, the daily increase of infected people is larger than the number of the daily reported new cases and can be represented by

$$
\dot{\hat{Z}}(t)=\delta(t) \dot{Z}(t) \quad \text { with } \quad \delta(t)>1 .
$$

The problem is that the exact values of $\delta(t)$ are not known - only more or less good estimates can be obtained by testing on small subgroups of the population. Because tests are central to fighting a pandemic, more and more of them are carried out over time, with the result that $\delta(t)$ will gradually shrink. However, this happens very slowly, so that $\delta$ can be treated as a constant over sufficiently short time intervals.

\subsection{General case}

For constant $\delta(t)=\delta_{0}$ follows $\hat{Z}(t)=\delta_{0} Z(t)$ from $\dot{\hat{Z}}(t)=\delta_{0} \dot{Z}(t)$, and after the replacement $Z(t) \rightarrow \delta_{0} Z(t)$ in Eqs. (4a) and (23a), the factors $\delta_{0}$ in the numerator and the denominator cancel each other out. This means that a constant relative number of unreported new cases leaves $A(t)$ and $R_{Z}(t)$ unchanged.

In the case of time-dependent $\delta=\delta(t)$, in Eqs. (4a) and (23a) $Z(t)$ must be replaced by $\hat{Z}(t)=\delta(t) Z(t)$. Even if $\delta(t)$ is not exactly known, one can investigate approaches for the temporal course of $\delta$ on the basis of conjectures in order to find out at least qualitatively how unreported cases affect the parameters $R_{Z}(t)$ and $A(t)$. This is done in the following section by means of a concrete example.

\subsection{Special case}

As a representative example, we examine the case

$$
Z(t)=70(1.1+\tanh (t / 100-3)) \quad \text { for } \quad 0 \leq t \leq t_{1}=600
$$

(the time being measured in days) in which the rate of new infections

$$
\dot{Z}(t)=7 /\left[10 \cosh ^{2}(t / 100-3)\right]
$$




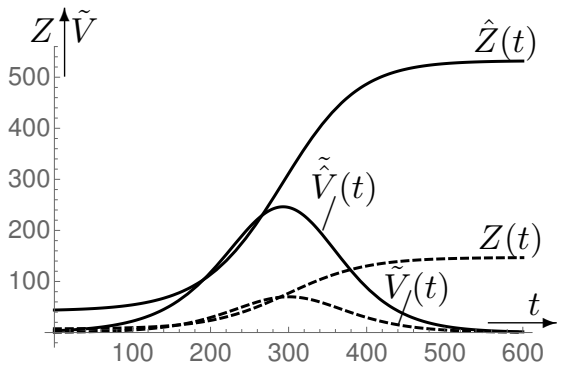

Fig. 3 The curves $Z(t)$ and $\tilde{V}_{Z}(t)=100 V_{Z}(t)$ for the wave-like time evolution according to Eqs. (36)-(37) are dashed, while the curves $\hat{Z}(t)$ and $\tilde{\hat{V}}_{Z}(t)=100 \hat{V}_{Z}(t)$, that include estimates for unreported cases, are solid. The enlargement from $V$ and $\tilde{V}$ to $\hat{V}$ and $\tilde{\hat{V}}$ was undertaken for making visible the course of the corresponding curves. (A logarithmic representation would have a similar effect but lead to an unusual image of $Z(t)$.)

represents the up and down of a wave-like time evolution (see Fig. 3). Concerning $\delta$ we set

$$
\delta(t)=5(1-t / 1000)
$$

which causes a decay from $\delta(0)=5$ for $t=0$ to $\delta(600)=2$ for $t=600$. Inserting this and $\dot{Z}(t)$ from Eq. (37) in Eq. (35) and integrating with respect to $t$ yields

$$
\hat{Z}(t)=6 Z(0)-G(0)+G(t)
$$

with

$$
G(t)=35 \ln \cosh (3-t / 100)+(7 / 20)(t-1000) \tanh (3-t / 100),
$$

where $\hat{Z}(0)=6 Z(0)$ was set as the initial condition. In Fig. 3, the functions $\hat{Z}(t)$ (solid) and $Z(t)$ (dashed) are shown together with their derivatives with respect to $t$, the corresponding parameter functions $\hat{A}(t)$ together with $A(t)$ and $\hat{R}_{Z}(t)$ together with $R_{Z}(t)$ are depicted in Fig. 4.
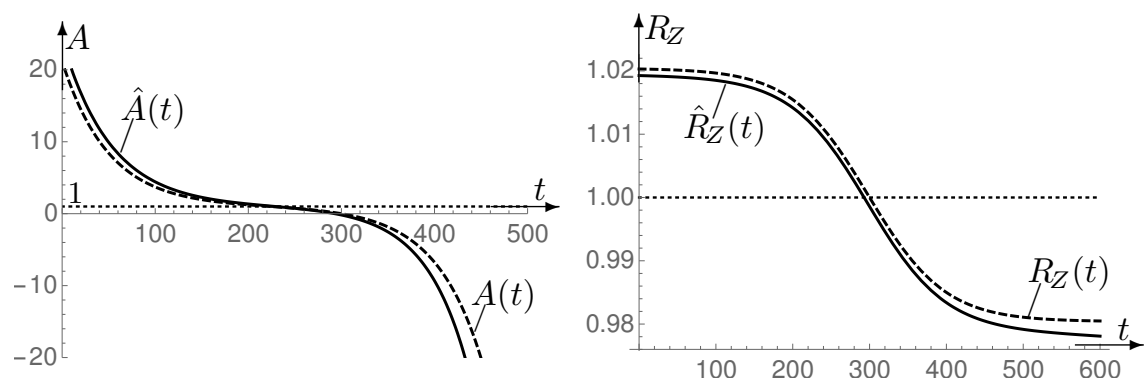

Fig. 4 Infection parameters $A(t), R_{Z}(t)$ and $\hat{A}(t), \hat{R}_{Z}(t)$ for the functions $Z(t)$ and $\hat{Z}(t)$, defined in Eqs. (36) and (39), and depicted in Fig. 3. Closer inspection shows that $R_{Z}(t)-\hat{R}_{Z}(t)$ and $A(t)-\hat{A}(t)$ are both monotonically increasing with $t$ whereby $A(t)-\hat{A}(t)$ starts with negative values and changes to positive values at $A=1$. 

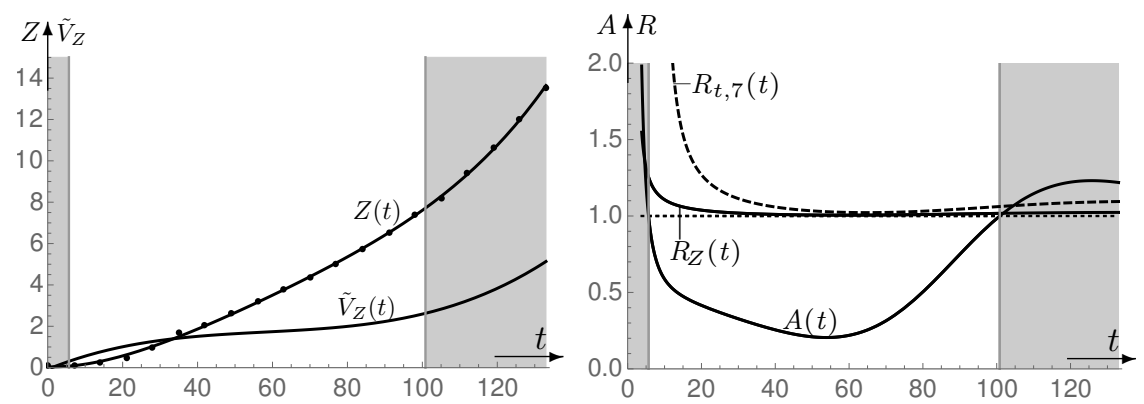

Fig. 5 Left diagram: Function $Z(t)$, derived from cumulative cases $Z_{n}$ every 7 days (represented by dots) for the world over a period of 130 days from March 4, 2020, and $\tilde{V}_{Z}(t)=20 V_{Z}(t)$ with $V_{Z}(t)=\dot{Z}(t), Z$ and $V_{Z}$ being counted in millions. Right diagram: Corresponding infection parameters $A(t), R_{Z}(t)$, and $R_{t, 7}$. The latter two come very close to limit 1 , but do not touch it. In the shaded areas, $Z(t)$ is super-exponential.

\section{Investigation of concrete covid-19 situations}

In this section, the course of the infection parameters $R_{Z}(t)$ and $A(t)$ is calculated for two specific examples and presented graphically. To do this, we use the reported data published by the Johns Hopkins Coronavirus Resource Center at the Johns Hopkins University (JHU) for the entire world [7], on the one hand over a period of 130 days at the beginning of the pandemic, and on the other hand over a period of 120 days in autumn 2020. We assume that the very large factor, by which $Z(t)$ should in fact be multiplied because of unreported cases (see Section 7.2), has not changed significantly within the selected time periods. In consequence, according to the explanations in Section $4, R_{Z}(t)$ and $A(t)$ can as well be calculated from the reported data with sufficient accuracy. The derivation of the function $Z(t)$ from this data takes place as set out at the beginning of Subsection 2.1. $R_{Z}(t)$ and $A(t)$ are then determined from $Z(t)$ using Eqs. (4) and (23).

\subsection{The world in spring 2020}

Fig. 5 shows in two diagrams the global progression of the pandemic in its initial phase from March 1st to July 3rd. In the left diagram the numbers $Z_{n}$ given by the JHU are represented by dots every 7 days; also shown are the function $Z(t)$ obtained from them by a best least-squares fit and the velocity $V_{Z}(t)=\dot{Z}(t)$. The diagram on the right shows the corresponding parameters $R_{Z}(t)$ and $A(t)$. The time intervals with $A>1$ and thus super-exponentially growing $Z(t)$ are shaded in both plots. $A(t)$ has a minimum just before $t=60$ and is fairly close to the value 0 , which indicates linear growth. Nevertheless, $Z(t)$ is increasing here much faster than in the left shaded area with super-exponential growth. This shows that the use of the word exponential to denote particularly dangerous growth can be misleading. Even in the shaded area on the right with super-exponential growth, the increase in $Z$ is not significantly faster than in the area with sub-exponential growth in front of it. From the right diagram it 


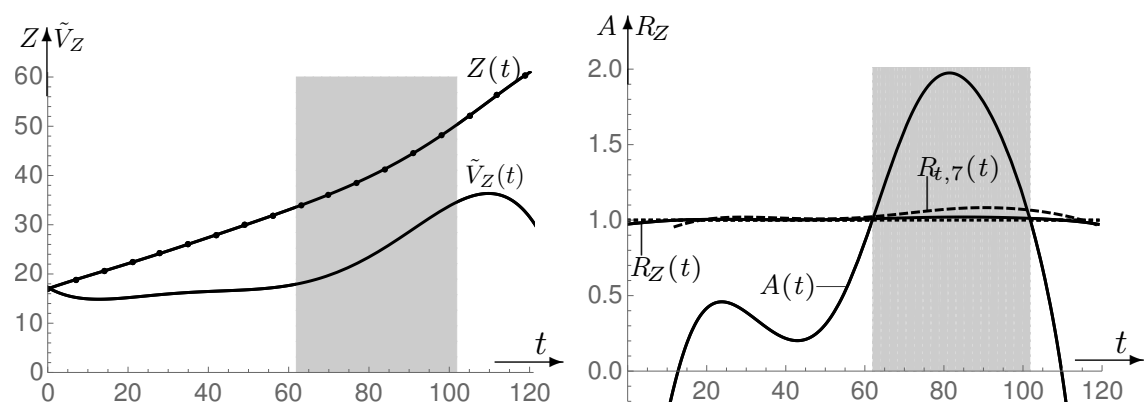

Fig. 6 The same applies here as in Fig. 5, with exception of the time interval, which extends over 120 days from July 29, 2020, and $\tilde{V}_{Z}=60 V_{Z}$. The parameters $R_{Z}(t)$ and $R_{t, 7}$ are shown again in Fig. 7, for better visibility in an enlarged view

can be seen that $A(t)$ reacts much more strongly to changes in $Z$ and $V_{Z}$ than $R_{Z}(t)$ and is thus more informative than $R_{Z}(t)$.

5.2 The world in autumn 2020

In Fig. 6 the same is shown as in Fig. 5, i.e. the global progression of the pandemic, but this time for a later phase in autumn 2020, extending over 120 days from July 29. Here the difference between $R_{Z}(t)$ and $A(t)$ becomes even clearer, while $R_{Z}(t)$ hardly changes at all compared to $A(t)$. For this reason, the courses of $R_{Z}(t)$ and $R_{t, \tau}$ are plotted again enlarged in Fig. 7. In this view, they even show similarities with that of $A(t)$. However, the minor changes of $R_{Z}(t)$ are not particularly reliable in view of the inaccuracies with regard to the values $Z_{n}$ from which $Z(t)$ and also $R_{Z}(t)$ are determined.

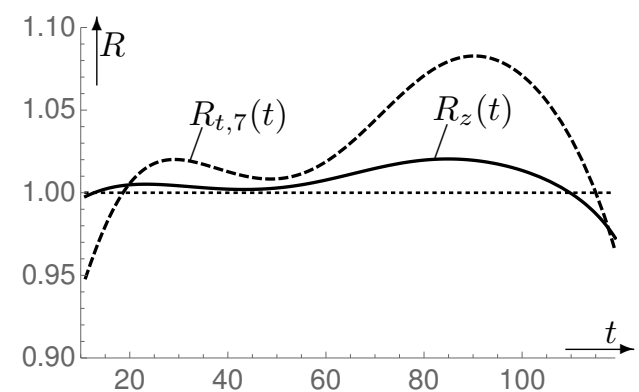

Fig. 7 Enlarged representation of the course of $R_{Z}(t)$ and $R_{t, \tau}$ from Fig. 6 


\section{Future projections by specifying $R_{Z}(t)$ or $A(t)$}

Of particular interest is how $Z(t)$ and $V_{Z}(t)$ advance when certain assumptions about the further course of $R_{Z}(t)$ or $A(t)$ are made.

Uniformly accelerated growth: In the case of constant acceleration we have

$$
\ddot{Z}(t)=a=\text { const }, \quad \dot{Z}(t)=V_{Z}(t)=V_{0}+a t, \quad Z(t)=Z_{0}+V_{0} t+a t^{2} / 2
$$

and

$$
R_{Z}(t)=\frac{\delta+t}{\delta+t-1}, \quad A(t)=\frac{Z_{0} / a+\delta t+t^{2} / 2}{(\delta+t)^{2}} \quad \text { with } \quad \delta=\frac{V_{0}}{a} .
$$

For $a>0$, from this follows $\delta>0, R_{Z}(0)=\delta /(\delta-1), A(0)=Z_{0} a / V_{0}^{2}$ and

$$
\frac{R_{Z}(t)}{R_{Z}(0)}=1-\frac{t}{\delta(\delta+t-1)} .
$$

If we now assume $R_{Z}(0)>0$, then it follows that $\delta>1, R_{Z}(0)>R_{Z}(t)>1$, and finally

$$
R_{Z}(t) \rightarrow 1 \quad \text { and } \quad A(t) \rightarrow \frac{1}{2} \quad \text { for } \quad t \rightarrow \infty
$$

In spite of the accelerated increase of $Z(t), R_{Z}$ decreases, and $\lim _{t \rightarrow \infty} A(t)<A(0)$ for $a>V_{0}^{2} /\left(2 Z_{0}\right)$.

Linear growth of $R_{Z}(t)$ : After the surprising result that $R_{Z}(t)$ decreases when $Z(t)$ is uniformly accelerated, we are interested under which circumstances $R_{Z}$ increases and examine, which consequences result from the ansatz

$$
R_{Z}(t)=R_{Z 0}+\alpha t \quad \text { with } \quad R_{Z 0}=R_{Z}(0) .
$$

With this we get from Eq. (4)

$$
\frac{\dot{V}_{Z}(t)}{V_{Z}(t)}=\frac{d \ln \left(V_{Z}(t) / V_{Z 0}\right)}{d t}=1-\frac{1}{R_{Z 0}+\alpha t}
$$

Integration with respect to $t$ results in $\ln \left(V_{Z}(t) / V_{Z 0}\right)=t-\ln \left(R_{Z 0}+\alpha t\right)^{1 / \alpha}$ or

$$
V_{Z}(t)=\frac{V_{Z 0} \mathrm{e}^{t}}{\left(R_{Z 0}+\alpha t\right)^{1 / \alpha}}=\frac{V_{Z 0} \mathrm{e}^{t}}{R_{Z}(t)} .
$$

$V_{Z}(t)$ grows slower than exponentially (sub-exponential growth) with increasing $R_{Z}(t)$ (positive $\alpha$ ) as well as decreasing $R_{Z}(t)$ (negative $\alpha$ ). 


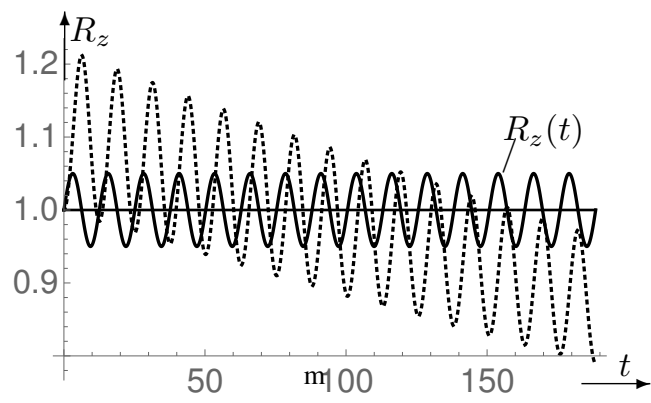

Fig. 8 The horizontal solid curve represents the function $R_{Z}(t)$ defined in Eq. (46). The dotted curve running from top left to bottom right represents the corresponding $V_{Z}(t) / V_{Z 0}$.

Arbitrary function $R_{Z}(t)$ : For given $R_{Z}(t)$, from Eq. (4) results the equation

$$
\dot{V}_{Z}(t) / V_{Z}(t)=1-1 / R_{Z}(t),
$$

which is solved by

$$
V_{Z}(t)=V_{Z 0} \mathrm{e}^{t-\int_{0}^{t}\left(1 / R_{Z}(\tau)\right) d \tau} \quad \text { for } \quad V_{Z}(0)=V_{Z 0},
$$

and integration of $\dot{Z}(t)=V_{Z}(t)$ yields

$$
Z(t)=Z_{0}+\int_{0}^{t} V_{Z}(\tau) d \tau
$$

with $V_{Z}(\tau)$ given by Eq. (44).

Oscillations of $R_{z}(t)$ around $R_{z}=1$ : In practice, at a lower level of newly infected individuals it is variously observed that the net reproduction number is close to 1 and alternately exceeds this value and falls below it. We simulate this situation by making the ansatz

$$
R_{Z}(t)=1+\varepsilon \sin (\omega t) .
$$

According to this and Eq. (44), the relative rate of new infections is

$$
\dot{Z}(t)=V_{Z}(t)=V_{Z 0} \mathrm{e}^{t-\int_{0}^{t}(1 /(1+\varepsilon \sin (\omega \tau)) d \tau} .
$$

The integral in the exponent can indeed be expressed through analytic functions,

$$
\int_{0}^{t} \frac{d \tau}{1+\varepsilon \sin (\omega \tau)}=\frac{2 \arctan \left(\frac{\varepsilon+\tan (\omega t / 2)}{\sqrt{1-\varepsilon^{2}}}\right)}{\omega \sqrt{1-\varepsilon^{2}}} .
$$

It is, however, rather tedious to get the various branches of the trigonometric functions aligned correctly adjacent to each other. We have therefore evaluated Eq. (47) numerically, and the result is shown in Fig. 8. The surprising outcome is that $V_{Z}(t)$ gradually decreases. The upward exceedings of limit 1 by $V_{Z}$ are therefore harmless if they are compensated for by undershoots of the same duration and strength. 
Growth with constant $A(t)=A$ : This case has already been dealt with in Subsection 3.1 for utilitarian purposes, but should be mentioned here because of its relevance for the present context.

\section{Footprint of $Z(t)$ in an $I_{\tau}$, A-plane}

\subsection{Cumulative incidence $I_{\tau}(t)$}

In addition to the effective reproductive number or related parameters such as our acceleration parameter $A(t)$, the cumulative incidence $I_{\tau}(t)$ also plays an important role in assessing the contamination of a population. It is defined as the fraction $N_{i}(t) / N$ of $N$ inhabitants that were infected within the last $\tau$ days, i.e.

$$
I_{\tau}(t)=N_{i}(t) / N
$$

The number $Z_{n}$ of newly infected individuals reported daily by the Coronavirus Resource Center of the JHU refers to the total population of a country with $Z_{P}$ inhabitants, and that also applies to our function $Z(t)$ derived from it. Summed up over the period from $t-\tau$ to $t$, the infection rate $\dot{Z}(t)$ yields a total of $Z(t)-Z(t-\tau)$ newly infected in relation to $Z_{P}$ inhabitants. With regard to the subset of $N$ inhabitants, this number has yet to be multiplied by the fraction $N / Z_{P}$ so that we finally get

$$
I_{\tau}(t)=\left(N / Z_{P}\right)[Z(t)-Z(t-\tau)] .
$$

In the covid-19 pandemic, the value $\tau=7$ has proven useful, and then $I_{7}(t)$ is often referred to as seven-day incidence. Concerning $N$ there is a preference for the value $10^{5}$. In the left diagram of Fig. $9, \hat{I}_{7}(t)$ is a best least square fit to discrete values $I_{7}\left(t_{n}\right)$ obtained from Eq. (48) for times $t_{n}$ in seven-day steps. The incidence can of course also be calculated with Eq. (48) from our fit function $Z(t)$ and is then denoted by $I_{\tau}(t)$.

There is a close relationship between the incidence $I_{\tau}(t)$ and the average growth velocity $\overline{V_{Z}(t)}$ of $Z(t)$ in the time interval $\tau$,

$$
\overline{V_{Z}(t)}=\frac{1}{\tau} \int_{t-\tau}^{t} V_{Z}(t) d t=\frac{1}{\tau} \int_{t-\tau}^{t} \dot{Z}(t) d t=\frac{Z(t)-Z(t-\tau)}{\tau} .
$$

From this and Eq. (48) we get

$$
I_{\tau}(t)=\left(\tau N / Z_{P}\right) \overline{V_{Z}(t)} .
$$

Because in our approach $V_{Z}(t)$ is calculated from the smoothed fit function $Z(t)$, for small $\tau$ the difference between $I_{\tau}(t)$ and $\tilde{V}_{Z}(t)=\left(\tau N / Z_{P}\right) V_{Z}(t)$ is only slight. This becomes also evident from the right diagram in Fig. 9, which compares the sevenday incidence $I_{7}(t)$ for the world $\left(N=10^{5}\right.$ and $\left.Z_{P}=7.804 \cdot 10^{9}\right)$ with the corresponding $\tilde{V}_{Z}(t)$ and with the $\hat{I}_{7}(t)$ from above over a period of 120 days in the fall of 2020 .

The effective reproduction number $R_{t, \tau}$ can be converted into a very simple form with the help of $I_{\tau}(t)$. From Eq. (48) follows $Z(t)-Z(t-\tau)=\left(Z_{P} / N\right) I_{\tau}(t)$, and if this is substituted into Eq. (22), the result is

$$
R_{t, \tau}=I_{\tau}(t) / I_{\tau}(t-4) .
$$




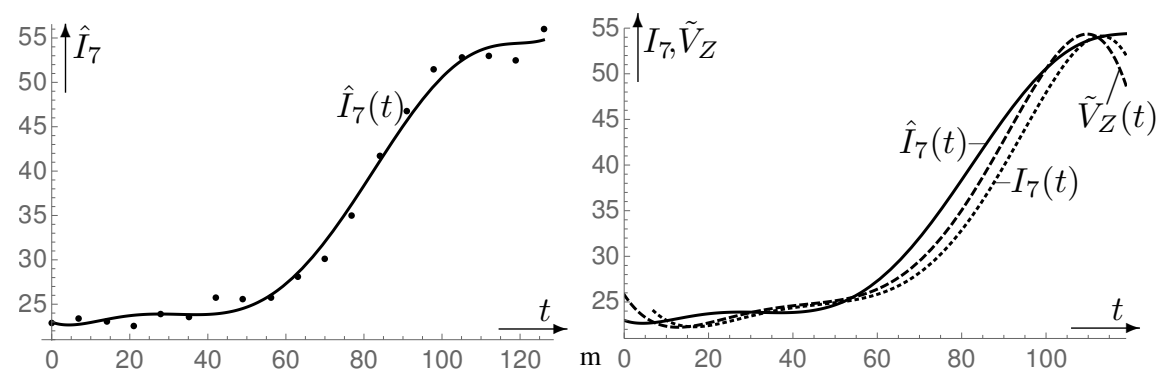

Fig. 9 Seven-day incidence for the world over a period of 120 days from July 29, 2020. In the left diagram $\hat{I}_{7}(t)$ can be seen along with the discrete values $I_{7}\left(t_{n}\right)$ to which it is adjusted. In the right diagram, $I_{7}(t), \hat{I}_{7}(t)$ and $\tilde{V}(t)$ are compared with each other. The differences are not great, but $\hat{I}_{7}(t)$ has the lowest volatility.

This equation has the important consequence that $R_{t, \tau}$ does not depend on the absolute, but only on the relative values of the two incidences: if both are multiplied by the same arbitrary factor, the result is the same value of $R_{t, \tau}$.

7.2 $A\left(I_{\tau}(t)\right)$ for the world and a wave-like time evolution of $V_{Z}(t)$

According to Eq. (50) $I_{\tau}(t)$ is a measure of the mean growth velocity of the infected individuals in a population, while $R_{Z}(t)$ or $A(t)$ are more suitable for inferences about the change of this velocity, i.e. the acceleration $\ddot{Z}(t)$. Taken alone, neither $I_{\tau}(t)$ nor $R_{Z}(t)$ or $A(t)$ are sufficient to assess the status of a pandemic. One can of course place two diagrams side by side for evaluation (e.g. the right diagram of Fig. 6 and the left diagram of Fig. 9), or one can even draw two curves in a single diagram, and professionals will draw the right conclusions from this. For a wider audience, viewing two diagrams in parallel is certainly less helpful. However, there is the possibility to combine both quantities in a single diagram with just one curve by plotting $\left\{I_{\tau}(t), A(t)\right\}$ as a time-dependent vector in an $I_{\tau}, A$ - plane. This procedure is also known as a parametric plot and provides a - possibly multi-valued - function $A\left(I_{\tau}\right)$.

This is demonstrated by means of two examples in Fig. 10. The left diagram shows the parametric plot $\left\{I_{7}(t), A(t)\right\}$ (or else the progression of $A\left(I_{7}(t)\right)$ in time) for the world over a period of 120 days in autumn 2020, using the same function $Z(t)$ as in Fig. 6. According to the WHO [8], the real number of infected people worldwide is about 20 times higher than the number of reported cases taken as a basis in the considered diagram. This means that $I_{7}(t)$ is about 20 times larger, and the curve $A\left(I_{7}(t)\right)$ has to be stretched by this factor in the direction of the $I_{7}$-axis, while the white area with its limit $I_{7}=50$ is left unchanged. The way back to the less dangerous white area is therefore much farther than it seems to be according to the diagram. Furthermore, the distance covered in the upper shaded area with large values $A$ is much longer than that in the area with smaller values $A$ but large $I_{7}$. 

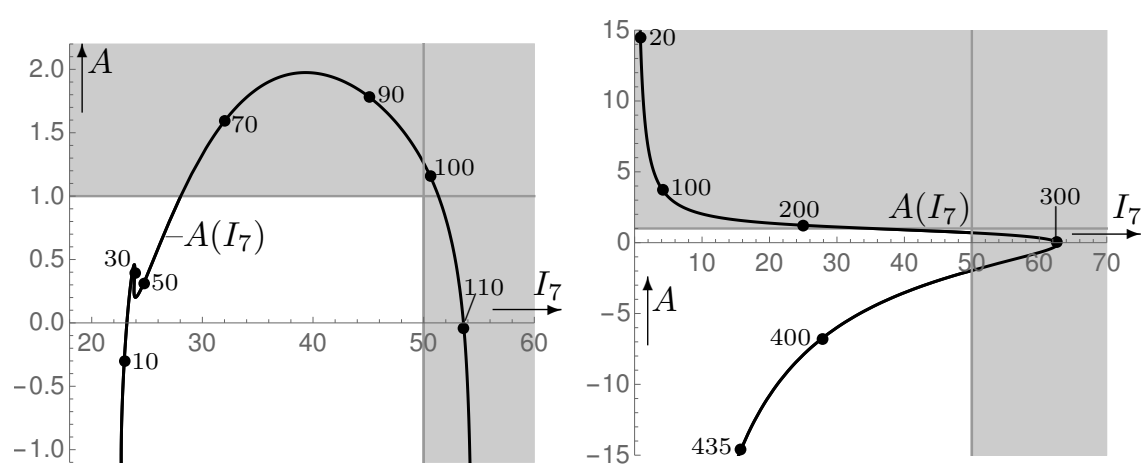

Fig. 10 Parametric plots $\left\{I_{7}(t), A(t)\right\}$ for the world in autumn 2020 (left diagram) and a hypothetical infection wave (right diagram), which are separately depicted in Fig. 6 and Figs. 3-4 respectively. The dots with small numbers next to them mark times measured in days

The parametric plot $\left\{I_{7}(t), A(t)\right\}$ for the hypothetical wave of infection from Eq. (36) can be seen in the right diagram of Fig. 10. The path into the white area with small values $I_{7}$ and negative values $A$ to be seen here is the one that must be aimed at in practice. Depending on where in the $I_{7}, A$-plane the current state is located, the primary objective must be to reduce either $I_{7}$ or $A$, or both simultaneously. In order to achieve this, however, one would have to know which measures are particularly effective for the respective goal.

Annotation: Diagrams of the type shown in Fig. 10 are also possible for $R_{Z}(t)$ and $R_{t, \tau}$.

\section{Summary and concluding remarks}

For our access to the epidemiological specialty of assessing the progression of a pandemic by suitable parameters such as a reproduction number, it was of decisive importance that the cumulative case numbers $Z_{n}$, assigned to discrete time steps $t_{n}$, were replaced by a continuously differentiable function $Z(t)$ with the help of a best least square fit. This made it possible to replace sums with integrals and differences with differentials, thus making the possibilities of differential and integral calculus accessible to us. In doing so, we were not only able to include a common effective reproduction number $R_{t, \tau}$, but also to introduce a number $R_{Z}$ that is similar to this, and can be expressed in a particularly simple and user-friendly manner in terms of $Z(t)$. Based on this, a further variable, referred as the acceleration parameter $A(t)$, was introduced, which is still more informative and facilitates a more differentiated characterization of the infection dynamics. In connection with the application of these parameters to specific situations, also the incidence $I_{\tau}(t)$ was finally introduced. It turned out particularly useful to bring $A(t)$ and $I_{\tau}(t)$ together into a single trajectory $A\left(I_{\tau}(t)\right)$ in a plane spanned by $I_{\tau}$ and $A$. 
To determine the function $Z(t)$, the program we used determines the best least square fit to the reported data $Z_{n}, t_{n}$ in the class of polynomials $Z(t)=\sum_{0}^{N} a_{n} t^{n}$. This gives good results with a relatively small number of sum terms, if the time period under consideration is not too long and the values $Z_{n}$ are not too volatile. If for $A(t)$ and $I_{\tau}(t)$ only the data from a short time interval (let's say the last 7-14 days) are used as in the determination of $R_{t, \tau}$, the fit in the class of polynomials should provide suitable results.

When examining the impact of unreported cases, it turned out that $R_{Z}(t)$ and $A(t)$ are not influenced if the factor $\delta$, by which $\dot{Z}(t)$ has to be multiplied to take them into account, remains constant. Even when it changes slowly, the two parameters are changed very little. However, this is different with the incidence which must be multiplied with $\delta$ even if the latter is constant.

In public discussion, the term exponential growth is often used for situations in which the number of new infections or their sum is increasing rapidly. Unfortunately, the real situation is much more tricky and difficult to communicate to the public. We have seen that the characterization of the infection process with the help of exponential functions is a comparison for which other functions could just as well be used. For the comparison to be meaningful, continuity conditions also have to be met. Because the growth rate $\dot{Z}(t)$ and the number $Z(t)$ of cumulative cases in parts behave differently, it still has to be specified to which of the two the characterization refers. It should also be noted that the comparison with an e-function is only possible locally; even for neighboring points in time, different e-functions must be used. The case that the behavior can be described by one and the same e-function over an extended period of time will virtually never occur. Furthermore, there are situations with accelerated growth in which the comparison with an e-function is not possible even locally. At the example of the world in spring 2020 (left diagram of Fig. 5) we saw that linear growth can be much faster than exponential growth. This exemplifies once more that the term exponential does not represent a complete and adequate characterization of growth, because there is slow and fast exponential growth ( $\mathrm{e}^{\alpha t}$ with a small or large $\alpha)$ as there can be slow and fast linear growth $(\dot{Z}(t)=\alpha$ with a small or large $\alpha)$.

As the Covid pandemic spread, at first only the effective reproduction number was used in the public discussion to assess the situation. It soon turned out that this was not sufficient for a complete characterization and has therefore been largely superseded by the incidence for some time now. From Eq. (51) it can be seen on the one hand how closely the two quantities are related, and on the other hand why neither of the two is sufficient on its own: $R_{t, \tau}$ has the same value for incidences of different sizes, e.g. $R_{t, \tau}=0.8=4 / 5=40 / 50$; on the other hand, the $R_{t, \tau}$-values indicate whether the incidences increase or decrease; especially $R_{Z}(t)$ and $A(t)$ provide a measure of the acceleration at which this happens. The $A\left(I_{7}(t)\right)$-graph in an $I_{7}, A$-plane introduced in Section 7.2 makes it possible in a simple way to combine $I_{7}(t)$ and $A(t)$ in a single trajectory. Roughly speaking, the diagram can be interpreted in such a way that the acceleration of the infection process is plotted against its velocity.

A question, the answer to which is important for the acceptance of restrictions that must be endured in order to contain the pandemic, is the following: Why must the restrictions still be maintained when the accelerated increase in new infections, often referred to as exponential growth, has been stopped by them and transformed 
into linear growth? A look at the left diagram of Figure 5 gives an answer: After the acceleration in the shaded area on the left, the growth rate $\dot{Z}(t)$ of new infections is higher than during the acceleration. If it is maintained, the situation is much worse than during the latter. This can also be seen from the diagrams in Figure 10. In the diagram on the left, an initial acceleration phase is followed by a deceleration phase, which after crossing the axis $A=0$ even turns into a negative acceleration. Due to the high number of unreported cases, the descending branch of trajectory $A\left(I_{7}(t)\right)$ is located much further to the right in the shaded area $I_{7} \geq 50$. The mean growth rate, proportional to $I_{7}$, must therefore be slowed down for a long time until it comes into the tolerable unshaded area with $I_{7}<50$ as it happens in the right diagram. Let a familiar analogy illustrate this effort. We consider a swimmer who wants to swim in a river to an upstream destination. Even to maintain his position relative to the banks requires quite some effort, and this must still be increased if he wants to move further upstream. Without effort, i.e. if he does nothing, he is even carried downstream by the flow, and the distance to his destination becomes larger and larger. In a similar way, measures must be maintained to prevent a further increase of the infection rate. In order to reduce it, the measures must even be tightened. In doing so it can make sense to replace very restrictive measures with others that are more targeted and less restrictive. However, it is imperative that they are just as effective.

\section{Appendix}

To show that the function $Z(t)$ from Eq. (27) changes for $A=1$ into $Z_{0} \mathrm{e}^{\alpha\left(t-t_{0}\right)}$ with $\alpha=V_{Z 0} / Z_{0}$, we set $A=1-\varepsilon$ whence

$$
Z(t)=Z_{0}\left[1+\varepsilon \alpha\left(t-t_{0}\right)\right]^{1 / \varepsilon}
$$

and let $\varepsilon$ go to zero. Using the fact that for small $\varepsilon$

$$
\mathrm{e}^{\varepsilon \alpha\left(t-t_{0}\right)}=1+\varepsilon \alpha\left(t-t_{0}\right)+\mathscr{O}\left(\varepsilon^{2}\right),
$$

we can write

$$
Z(t)=Z_{0}\left[\mathrm{e}^{\varepsilon \alpha\left(t-t_{0}\right)}-\mathscr{O}\left(\varepsilon^{2}\right)\right]^{1 / \varepsilon}
$$

and get

$$
Z(t) \rightarrow Z_{0} \mathrm{e}^{\alpha\left(t-t_{0}\right)} \quad \text { for } \quad \varepsilon \rightarrow 0
$$

\section{Appendix}

Eq. (25) also applies for time-dependent $A(t)$ and can be converted into

$$
\frac{d}{d t} \ln \frac{V_{Z}(t)}{V_{Z 0}}=\frac{d}{d t}\left(A(t) \ln \frac{Z(t)}{Z_{0}}\right)-\dot{A}(t) \ln \frac{Z(t)}{Z_{0}}
$$


in this case. Integration with respect to $t$ yields

$$
V_{Z}(t) / V_{Z 0}=\left(Z(t) / Z_{0}\right)^{A(t)}-\int_{t_{0}}^{t} J(\tau) d \tau \quad \text { with } \quad J(\tau)=\dot{A}(\tau) \ln \left(Z(\tau) / Z_{0}\right) .
$$

For $t=t_{0}+\delta t$ with $\delta t / t_{0} \ll 1$ we get

$$
\int_{t_{0}}^{t} J(\tau) d \tau=J\left(t_{0}\right) \delta t=0
$$

because $J\left(t_{0}\right)=\dot{A}\left(t_{0}\right) \ln 1=0$. This shows that Eq. (28) and thus also Eq. (29) derived from it also apply locally for time-dependent $A$.

Acknowledgements: For calculations and plots MATHEMATICA 10 was used.

\section{Declarations}

Funding: The work was performed without any funding.

Financial interests: none

Non-financial interests: none

Ethics approval: appears unnecessary for the following reason: The work is strongly mathematical, and personal data are purely statistical in nature and freely accessible to everyone.

Conflicts of interest: Numerical calculations and their graphic representation were carried out with MATHEMATICA. This is listed in an acknowledgment and also through entries in the bibliography.

\section{References}

1. Robert Koch-Institut, Erläuterung der Schätzung der zeitlich variierenden Reproduktionszahl R, p. 2 (2020)

2. Robert Koch-Institut, Erläuterung der Schätzung der zeitlich variierenden Reproduktionszahl R, p. 3 (2020)

3. Cori A, Ferguson NM, Fraser C, and Cauchemez S, "A New Framework and Software to Estimate Time-Varying Reproduction Numbers During Epidemics", American journal of epidemiology 178(9), 1505-1512 (2013) https://doi.org/10.1093/aje/

4. https://en.wikipedia.org/wiki/Basic_reproduction_number

5. Wolfram S., Mathematica: A System for Doing Mathematics by Computer, Addison-Wesley Publishing Company, Redwood City Cal., p. 542 (1988)

6. Wolfram S., Mathematica: A System for Doing Mathematics by Computer, Addison-Wesley Publishing Company, Redwood City Cal., p. 461 (1988)

7. https://coronavirus.jhu.edu/map.html

8. https://apnews.com/article/virus-outbreak- archive-united-nations-54a3a5869c9ae4ee623497691e796083 

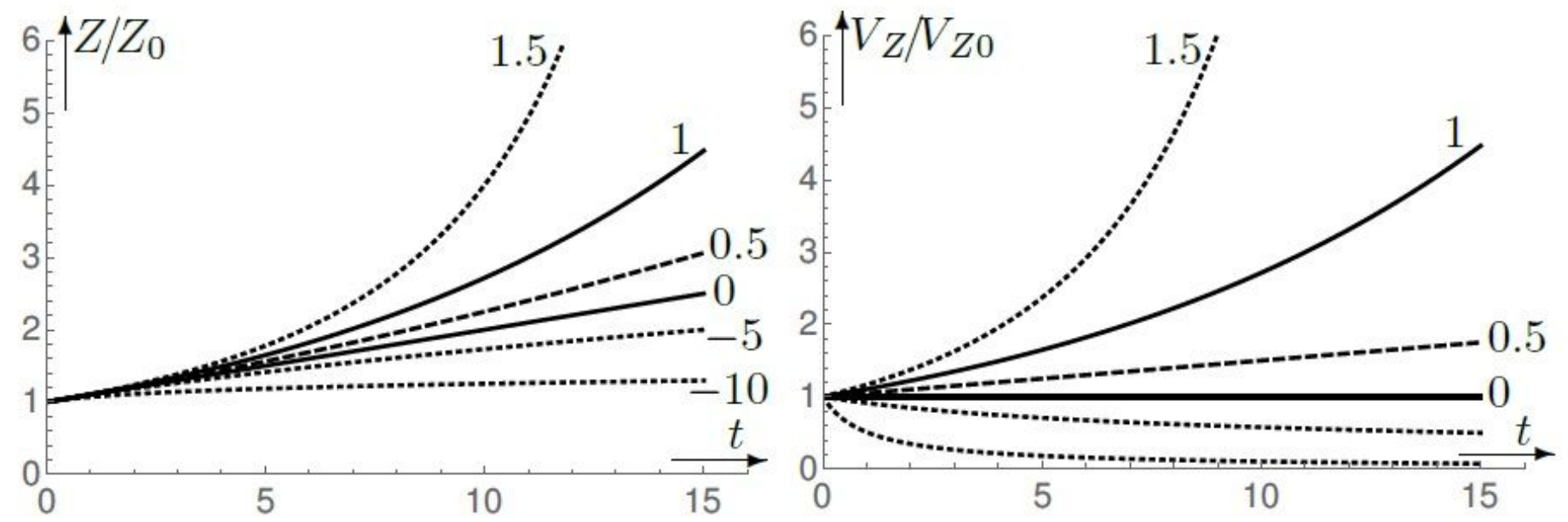

\section{Figure 1}

The left diagram shows $Z(t)=Z 0$ and the right diagram $V Z(t)=V Z 0$ for $A=1: 5 ; 1 ; 0: 5 ; 0 ;-5 ;-10$ in the order from top to bottom. The curves $A=1$ for exponential growth and $A=0$ for linear growth are solid. The dashed curve $A=0: 5$ can (but must not) be considered as the boundary between super-linear and subexponential.
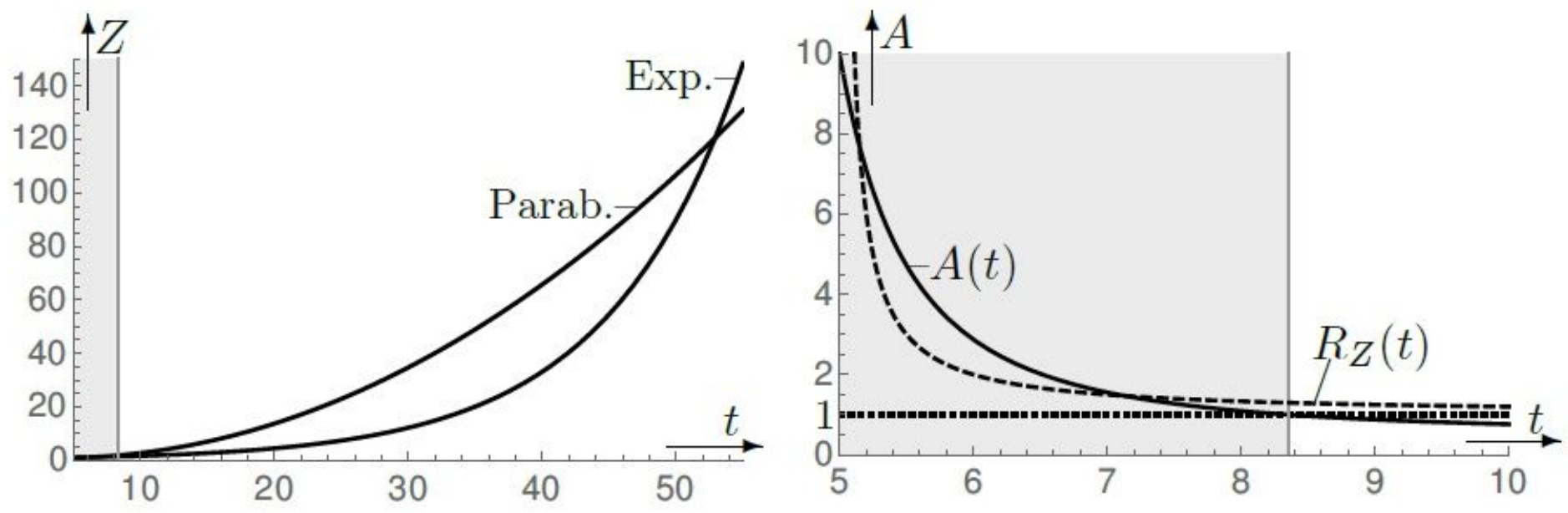

Figure 2

The parabola and exponential function shown in the diagram on the left have the same function value and the same slope but different curvature at $t=0$. As a result, the parabola has higher values $Z(t)$ than the exponential function over a longer period of time. In the diagram on the right, the values $A(t)$ and $R Z(t)$ belonging to the parabola are plotted, with the super- exponential area being shaded. 


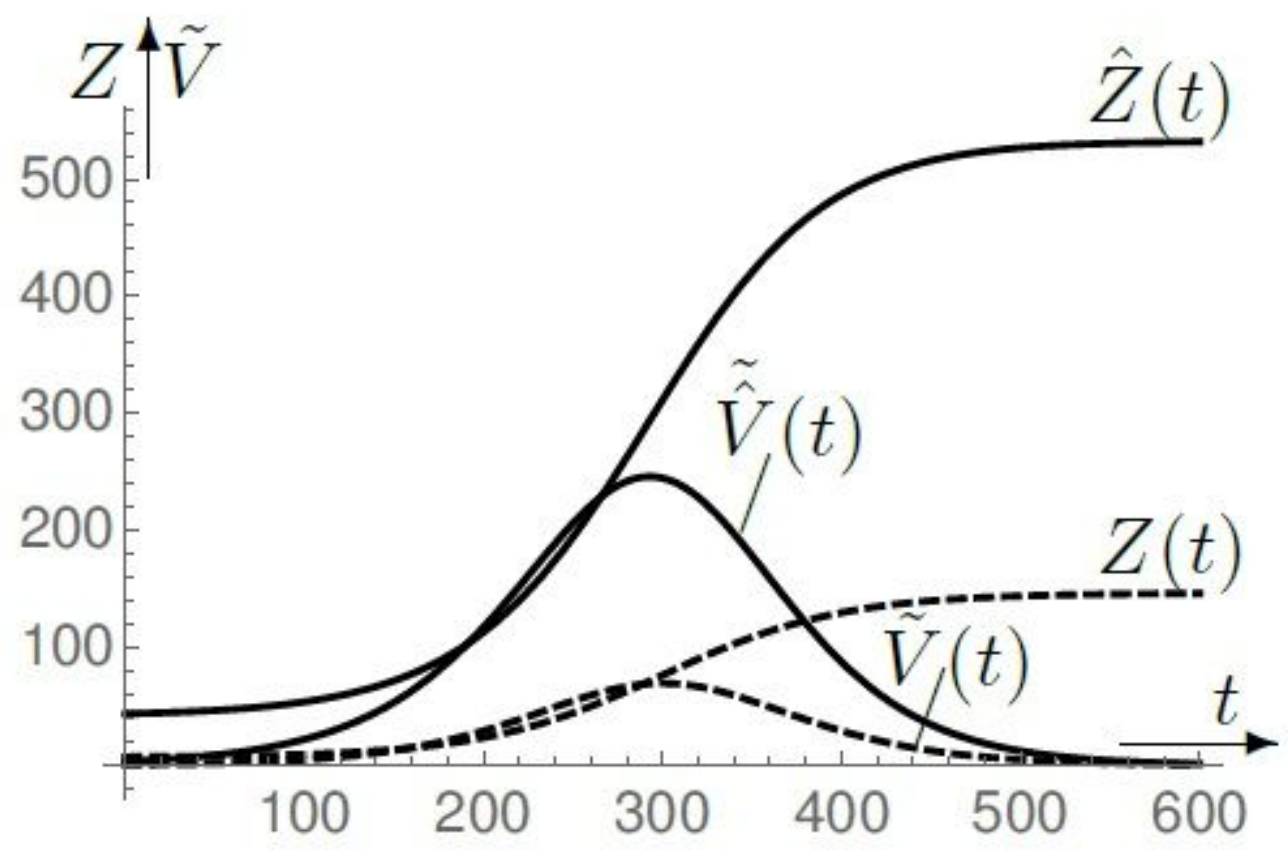

Figure 3

The curves $Z(t)$ and $\sim \mathrm{VZ}(\mathrm{t})=100 \mathrm{VZ}(\mathrm{t})$ for the wave-like time evolution according to Eqs. (36)-(37) are dashed, while the curves ${ }^{\wedge} Z(t)$ and ${ }^{\sim} \vee V Z(t)=100^{\wedge} V Z(t)$, that include estimates for unreported cases, are solid. The enlargement from $V$ and $\sim \mathrm{V}$ to ${ }^{\wedge} \mathrm{V}$ and ${ }^{\sim}{ }^{\wedge} \mathrm{V}$ was undertaken for making visible the course of the corresponding curves. (A logarithmic representation would have a similar effect but lead to an unusual image of $Z(t)$.)
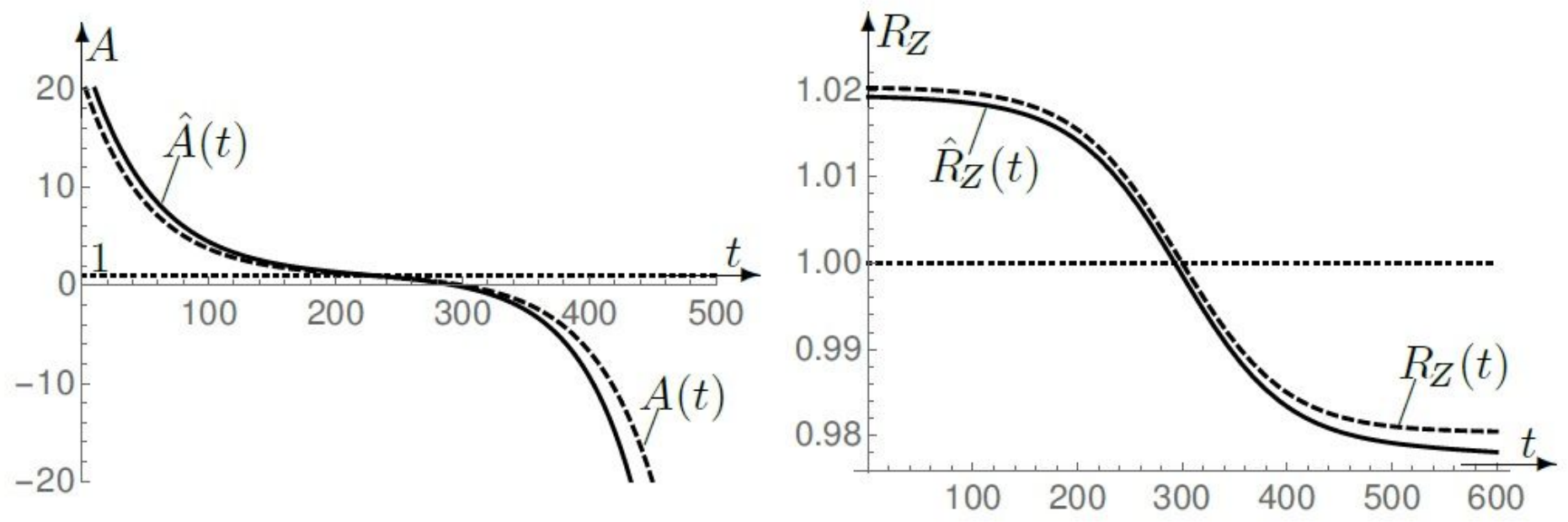

Figure 4

Infection parameters $A(t), R Z(t)$ and ${ }^{\wedge} A(t)$, ${ }^{\wedge} R Z(t)$ for the functions $Z(t)$ and ${ }^{\wedge} Z(t)$, defined in Eqs. (36) and (39), and depicted in Fig. 3. Closer inspection shows that $R Z(t)-{ }^{\wedge} R Z(t)$ and $A(t)-{ }^{\wedge} A(t)$ are both monotonically increasing with t whereby $A(t)-{ }^{\wedge} A(t)$ starts with negative values and changes to positive values at $A=1$. 

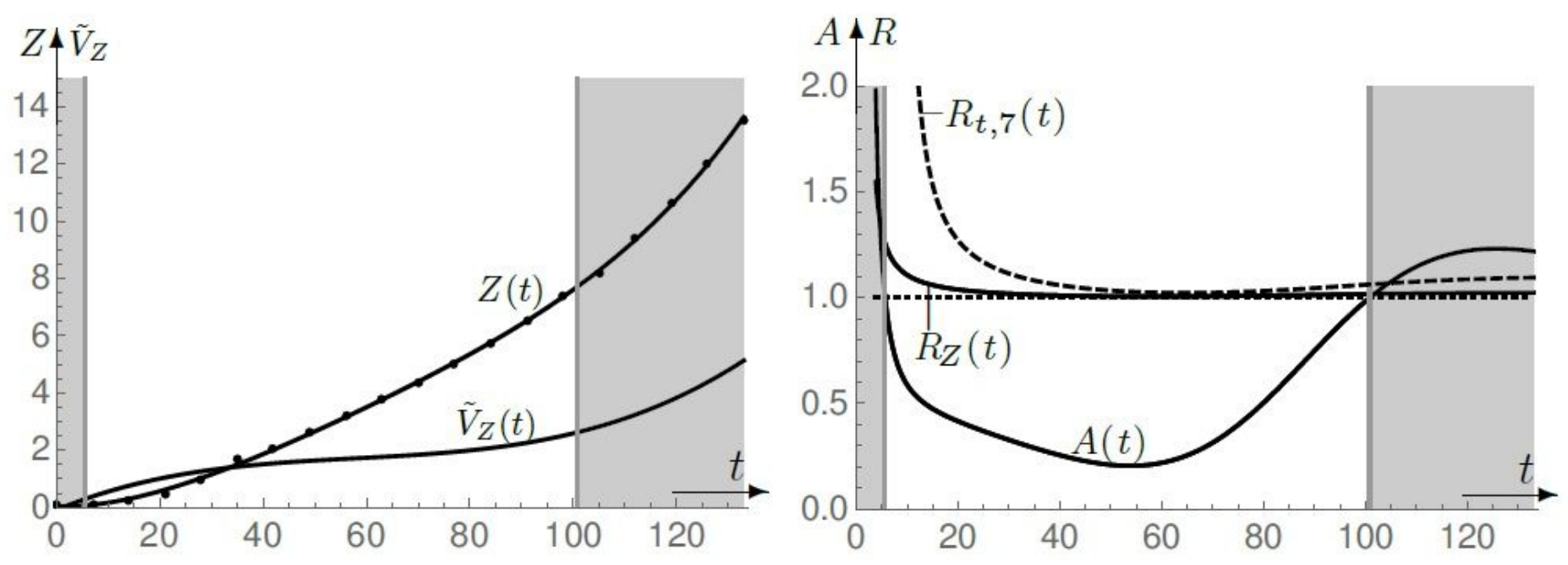

\section{Figure 5}

Left diagram: Function $Z(t)$, derived from cumulative cases $Z n$ every 7 days (represented by dots) for the world over a period of 130 days from March 4, 2020, and $\sim \mathrm{VZ}(\mathrm{t})=20 \mathrm{VZ}(\mathrm{t})$ withVZ $(\mathrm{t})=\mathrm{Z} Z(\mathrm{t}), \mathrm{Z}$ andVZ being counted in millions. Right diagram: Corresponding infection parameters $A(t), R Z(t)$, and Rt;7. The latter two come very close to limit 1 , but do not touch it. In the shaded areas, $Z(t)$ is super-exponential.
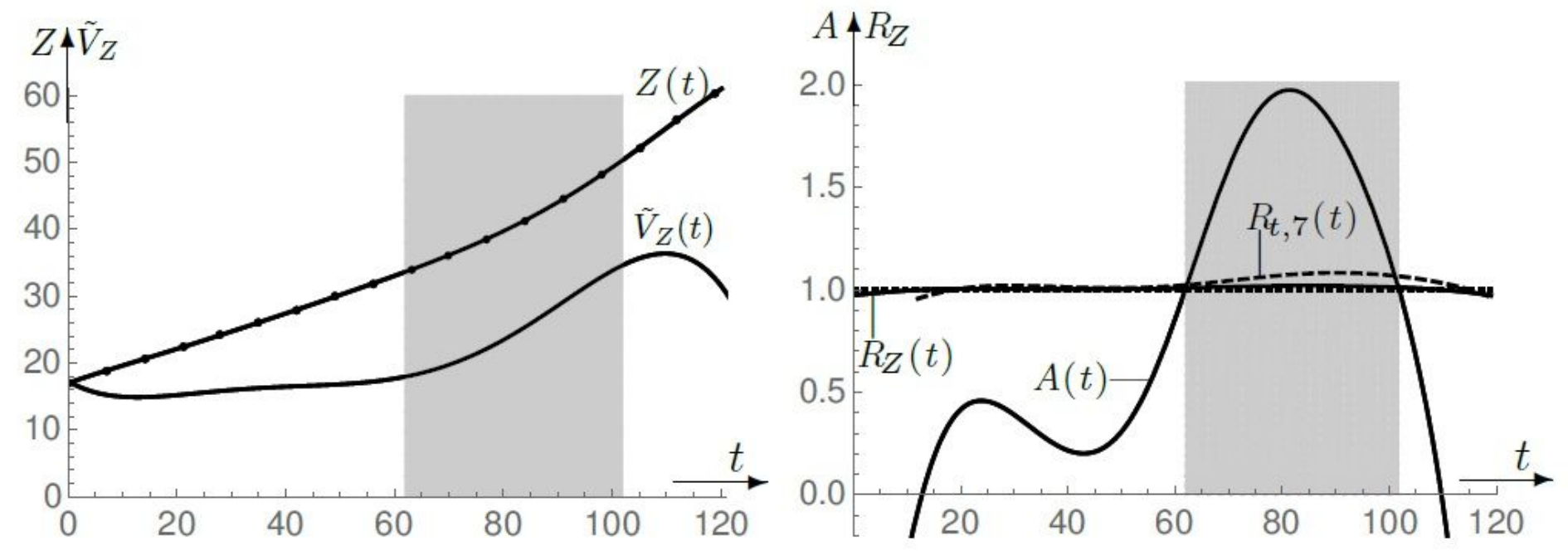

Figure 6

The same applies here as in Fig. 5, with exception of the time interval, which extends over 120 days from July 29, 2020, and VZ=60VZ. The parameters RZ(t) and Rt;7 are shown again in Fig. 7, for better visibility in an enlarged view 


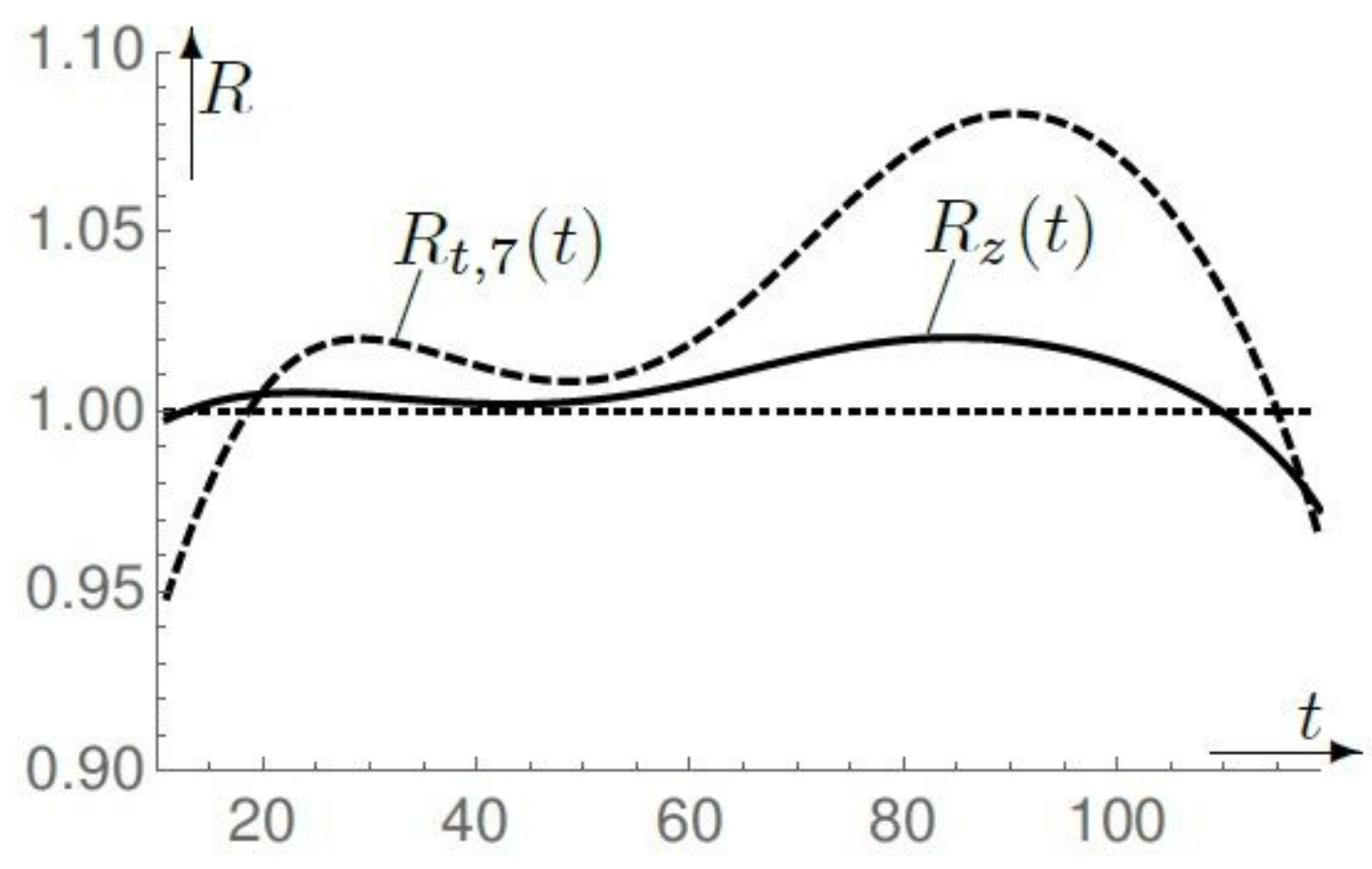

Figure 7

Enlarged representation of the course of RZ(t) and Rt,t from Fig. 6

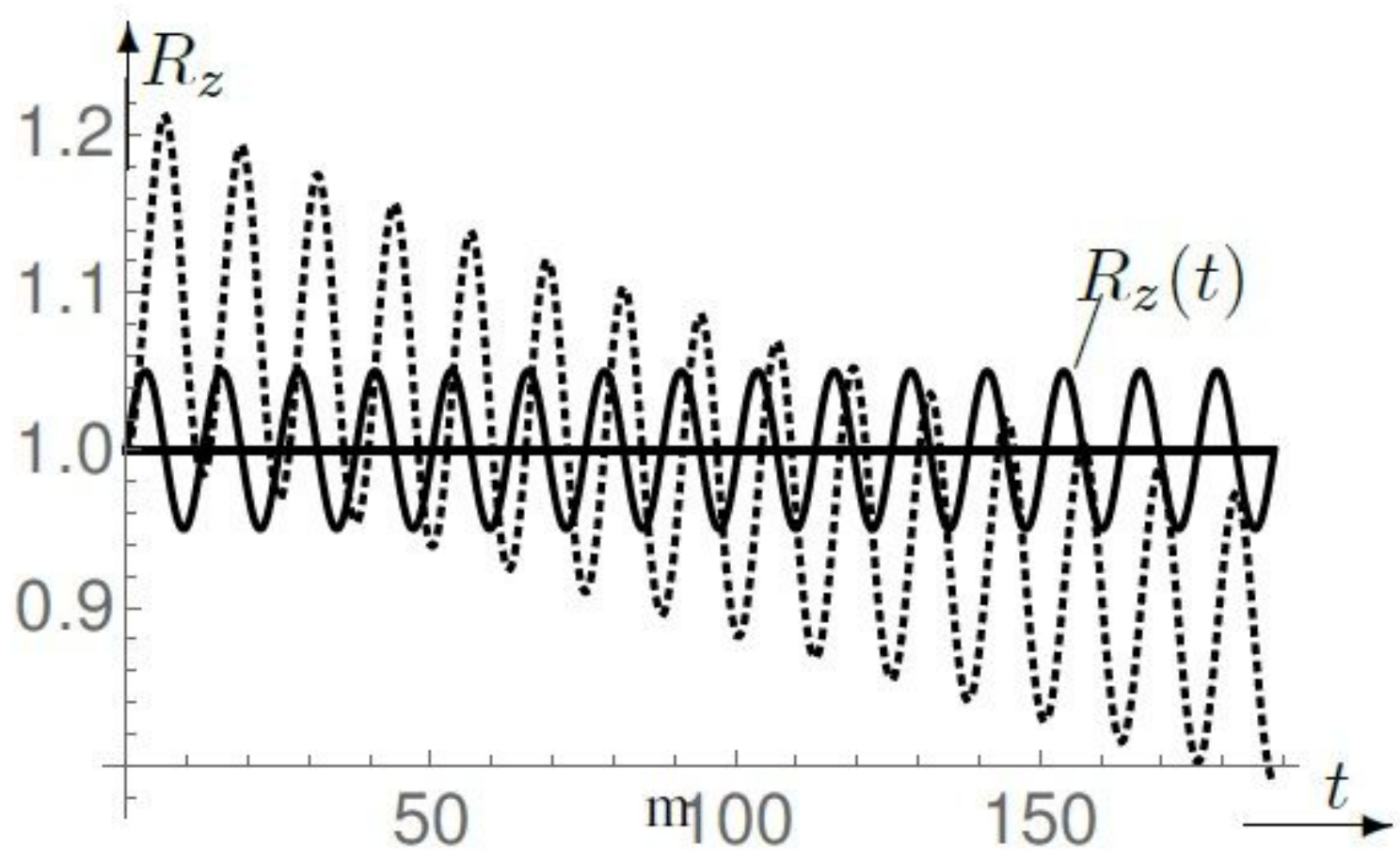

Figure 8

The horizontal solid curve represents the function RZ(t) defined in Eq. (46). The dotted curve running from top left to bottom right represents the corresponding $\mathrm{VZ}(\mathrm{t})=\mathrm{VZO}$. 


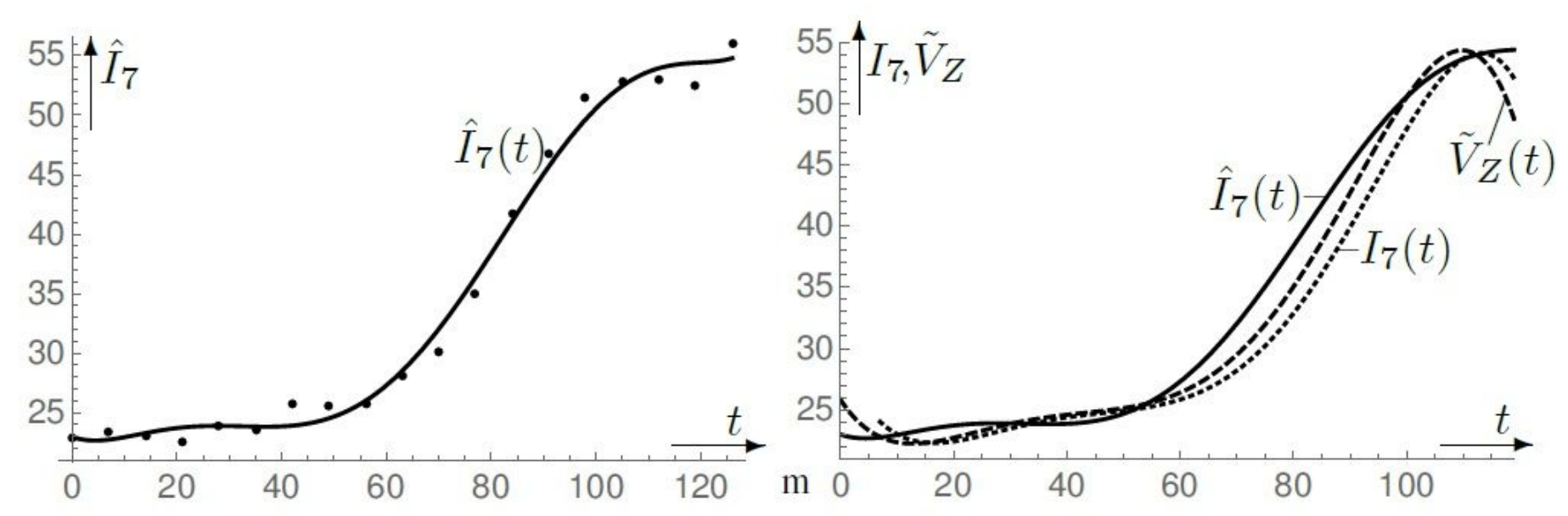

Figure 9

Seven-day incidence for the world over a period of 120 days from July 29, 2020. In the left diagram ^ 17(t) can be seen along with the discrete values $17(\mathrm{tn})$ to which it is adjusted. In the right diagram, $17(\mathrm{t}),{ }^{\wedge} 17(\mathrm{t})$ and $\sim \mathrm{V}(\mathrm{t})$ are compared with each other. The differences are not great, but ${ }^{\wedge} 17(\mathrm{t})$ has the lowest volatility.
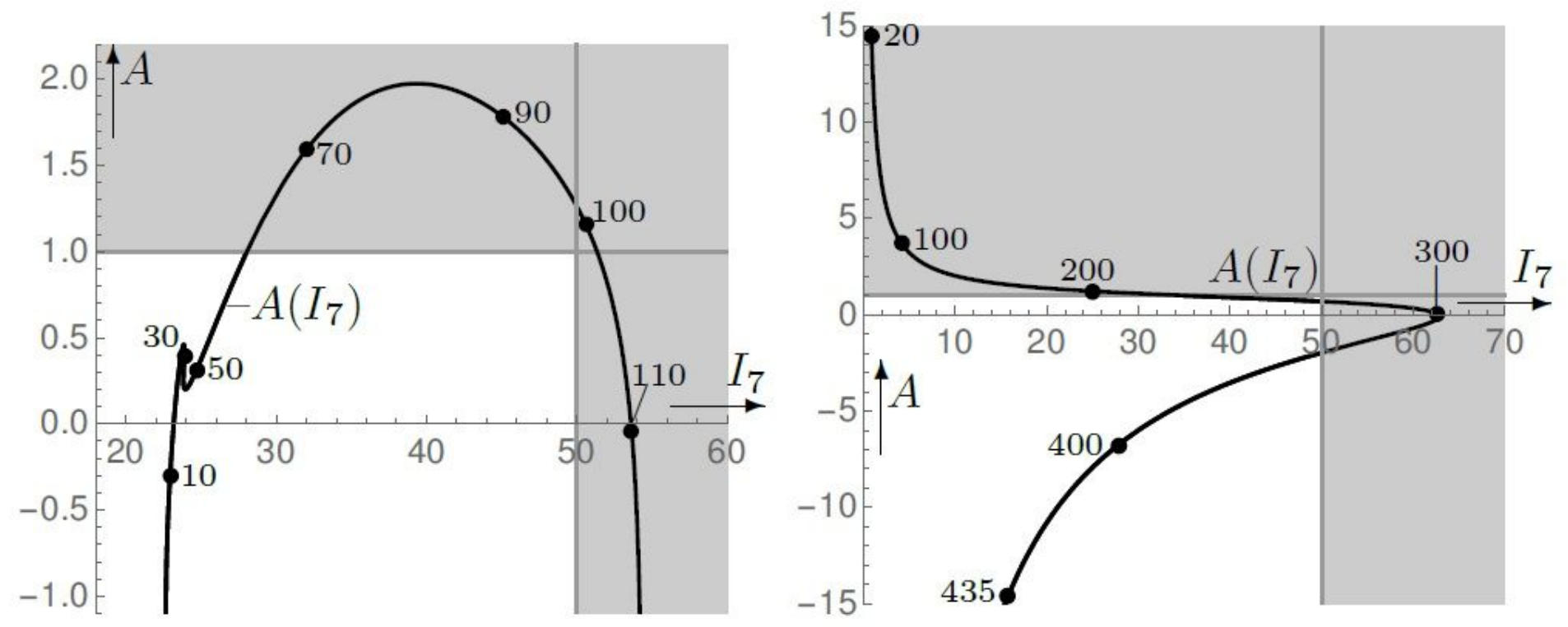

Figure 10

Parametric plots $\mathrm{fl} / \mathrm{t}(\mathrm{t}) ; \mathrm{A}(\mathrm{t}) \mathrm{g}$ for the world in autumn 2020 (left diagram) and a hypothetical infection wave (right diagram), which are separately depicted in Fig. 6 and Figs. 3-4 respectively. The dots with small numbers next to them mark times measured in days 